\title{
Intensive Care Management of the Neuromuscular Patient
}

\author{
Swarna Rajagopalan, Sanam Baghshomali, \\ Jovany Cruz Navarro, and Atul A. Kalanuria
}

\subsection{Introduction}

Clinically, neuromuscular conditions are diseases of the nervous system that affect the motor neuron unit between the anterior horn cells and the muscle. This group of diseases classically affects lower motor neuronal body which could be in the cranial nerve nuclei or spinal cord, the corresponding axon including its multiple branches, and the neuromuscular junction. Patients can have symptoms due to affection of any part of the motor neuron unit thus making this group of diseases quite heterogenous. Patients presenting acutely can have a range of symptoms. While some may require observation only, others could have lifethreatening complications. Occasionally, these conditions can have an acute presentation that

S. Rajagopalan $\cdot$ S. Baghshomali

Department of Neurology and Neurosurgery,

West Virginia University, Morgantown, WV, USA

J. C. Navarro

Department of Anesthesiology and Neurosurgery, Baylor College of Medicine, Houston, TX, USA

\footnotetext{
A. A. Kalanuria $(\bowtie)$

Department of Neurology, The Hospital of the University of Pennsylvania, Philadelphia, PA, USA

e-mail: Atul.Kalanuria@upenn.edu
}

necessitates admission to an intensive care unit. Patients suffering from acute non-traumatic neuromuscular weakness typically present with difficulties in protecting their airway with or without symptoms of respiratory distress. Certain neuromuscular diseases can also lead to dysfunction of the autonomic nervous system which can lead to circulatory collapse, while others can be due to a consequence of prolonged critical illness. When respiratory symptoms do develop, these patients often require ventilation support with the use of invasive or noninvasive devices.

\subsection{Clinical Presentations and Localization of Neuromuscular Disorders (NMD)}

The two crucial steps in the diagnosis and management of neuromuscular diseases are:

1. Identifying the anatomic localization of the site of the lesion that is producing symptomology

2. Determining the etiology of the lesion

A systematic approach to localization is to place the lesion within the nervous system. Disease can occur in the central nervous system (CNS), containing the brain and spinal cord, or peripheral nervous system (PNS). The CNS includes the cerebral motor cortex, corticospinal tracts, and spinal cord, and the PNS includes 
spinal nerves, nerve roots, nerve branches, neuromuscular junctions (NMJ), and muscles [1]. CNS and PNS lesions should be identifiable from a detailed neurologic examination. The distribution of motor weakness, presence of sensory involvement, presence or absence of deep tendon reflexes, and pain are all important in localizing the lesion in the nervous system. These features are listed for neuromuscular diseases that are commonly encountered in critical care (Table 5.1). The focus of the rest of this chapter will be to discuss neuromuscular diseases of the PNS in greater detail.

\subsection{Sites of Neuromuscular Disease}

- $C N S$-diseases affecting the brain and spinal cord result in upper motor neuron (UMN) impairment. These include trauma, infections like abscesses, inflammatory diseases like multiple sclerosis or acute disseminated encephalomyelitis (ADEM), tumors, degenerative diseases, vascular disease like stroke, toxicities like carbon monoxide poisoning, genetic diseases like leukodystrophies, and metabolic diseases like vitamin deficiencies. Appropriate imaging studies of the CNS and PNS based on the suspected site like computed tomography (CT), magnetic resonance imaging (MRI), or plain radiographs and perhaps a cerebrospinal fluid (CSF) examination may be necessary to identify the primary disease.

- Anterior horn cells-diseases affecting anterior horn cells include degenerative diseases like amyotrophic lateral sclerosis (ALS) and familial spinal atrophy, infections like poliomyelitis and West Nile virus as well as toxins like lead poisoning. Patient age, exposure history, family history, time course of disease, laboratory tests, nerve conduction studies, and CSF examination can help differentiate between these diseases.

- Spinal nerve roots-diseases causing acute radiculopathies include trauma resulting in spinal disk herniation, infections like Lyme disease and varicella zoster virus, inflammatory diseases like acute inflammatory demyelinating polyradiculoneuropathy (AIDP; Guillain-Barré syndrome), neoplasms including lymphoma or multiple myeloma, as well as vascular diseases like arteriovenous malformations and vasculitis.

- Peripheral spinal nerves and branchesdiseases can affect a single nerve, for instance, as a sequelae of nerve compression as in carpal tunnel syndrome, or multiple nerves. Mononeuropathy multiplex, an ischemic disease affecting multiple nerves, can occur as a result of diabetes mellitus (DM) or vasculitis. A symmetric polyneuropathy with weakness and sensory symptoms has a wide variety of causes, ranging from common such as DM, critical illness, HIV, and alcohol abuse, less common such as vitamin B12 deficiency and Lyme disease, to rare such as porphyria and Charcot-Marie-Tooth disease. Symmetric polyneuropathy often occurs as an adverse effect of medication or as a manifestation of systemic disease. The characteristics (axonal or demyelinating) and rate of progression of disease can help differentiate between causes.

- Neuromuscular junction (NMJ)—diseases of neuromuscular transmission that affect the presynaptic junction can interfere with acetylcholine (ACh) release as in botulism or affect voltage-gated presynaptic calcium channels as in Lambert-Eaton syndrome. Other less common presynaptic diseases include congenital myasthenic syndromes, severe hypermagnesemia, and tick paralysis. Diseases that affect the postsynaptic junction include the generation of anti-acetylcholine receptor antibodies in myasthenia gravis and inhibition of acetylcholinesterase by organophosphate poisoning.

- Muscles - diseases of the muscle have a wide variety of causes, including inflammatory disorders such as critical illness and polymyositis, infections such as influenza, endocrinopathies such as hypothyroidism, metabolic myopathies such as hypokalemia, drugs and toxins such as corticosteroids or statins, and the various causes of rhabdomyolysis. The specific diagnosis may be suspected from the history and course of disease. The presence of features of a systemic 
5 Intensive Care Management of the Neuromuscular Patient

65

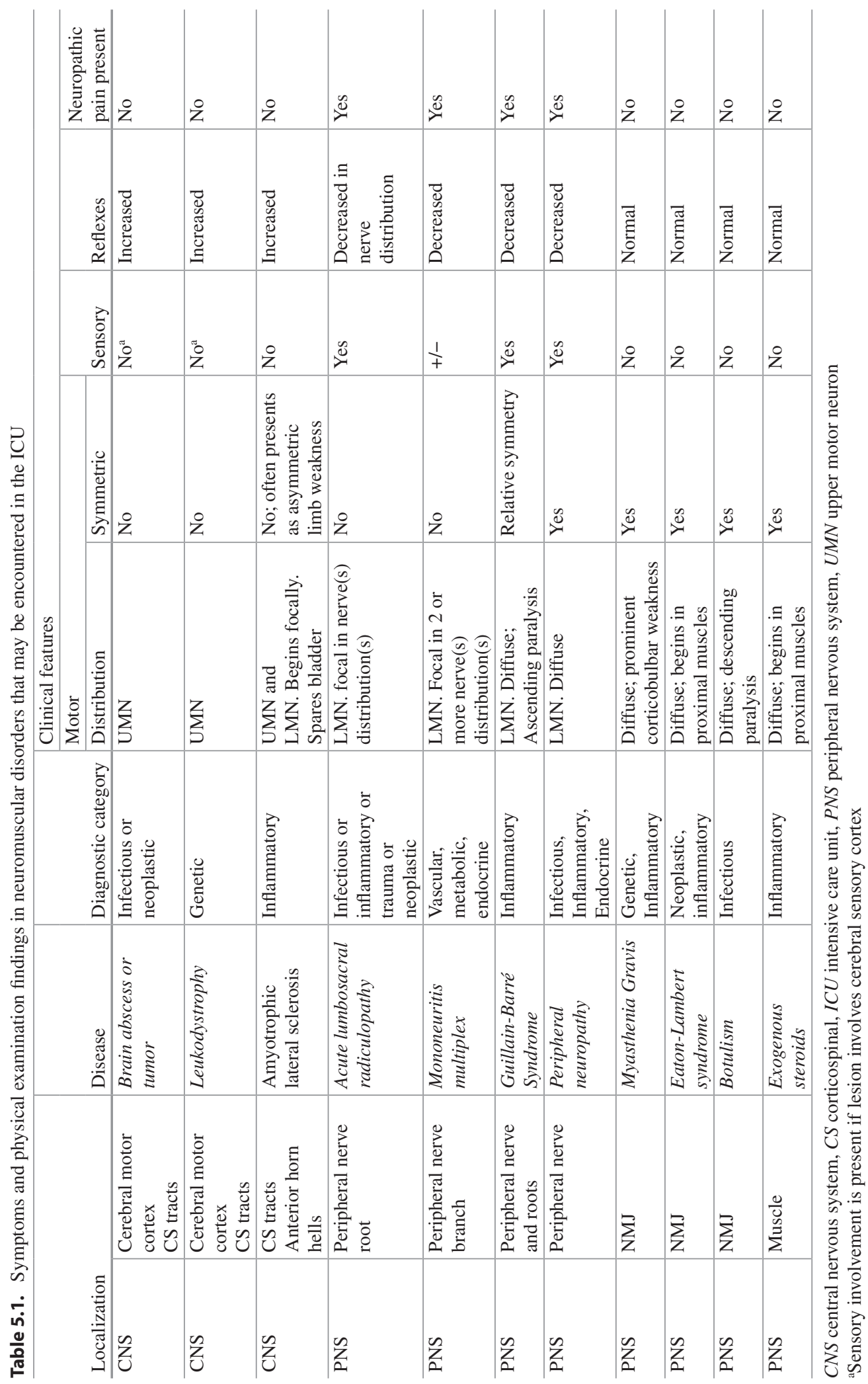


illness such as systemic lupus erythematosus may be indicative of myositis. Medication, alcohol, or substance abuse may be a clue to drug-induced myopathy. History of endocrinopathies such as thyroid dysfunction could be a clue to diagnosis. A history of recurrent episodes of exertion-related hematuria and weakness could suggest a metabolic myopathy.

Distribution of motor muscle weakness as a guide to localization of lesion-Determining the pattern of weakness is diagnostically important and can localize the lesion to the CNS or PNS. Weakness of both CNS and PNS can be broadly classified as diffuse, involving all muscle groups in varying extents or focal, involving specific distributions of the brain, nerve, or muscle. Diffuse muscle weakness can involve muscles in a symmetric pattern or asymmetric pattern. Symmetric weakness can cause proximal or distal loss of muscle strength. As such, in patients with diffuse weakness, the physician should determine whether the loss of function is proximal or distal while obtaining patient history by asking which physical activities muscle weakness limits as well as by conducting a focused neurologic examination testing specific muscle group strength against resistance.

Proximal muscle weakness involves the axial muscle groups, deltoids, and hip flexors. If the patient has difficulty lifting their head off the bed (neck flexors), rising from a seated position (quadriceps), or brushing his or her hair (deltoids), the weakness is proximal. Proximal muscle weakness is typically seen in the various myopathies, certain muscular dystrophies, and myasthenia gravis.

Distal muscle weakness is characterized by decreased grip strength, weakness of wrist flexion or extension, decreased plantar flexion strength, and foot drop. If the patient has difficulty walking on their heels (tibialis anterior, extensor hallucis longus) and toes (gastrocnemius/soleus muscles) or buttoning their shirt with their hands (intrinsic muscles), the muscle weakness is distal. Distal symmetric weakness can be a characteristic of early motor neuron disease or peripheral neuropathy.

Determining etiology of lesion-Once the neuromuscular site of the lesion has been localized, the etiology of disorders at each site can be categorized as trauma, infectious, inflammatory, neoplastic, degenerative, vascular, toxic, or metabolic in origin (see above sites of neuromuscular disease for examples). Once anatomic localization is made, it is pieced together with patient history to generate differential diagnoses.

\subsection{Clinical Investigations}

\subsubsection{Laboratory Tests}

Basic laboratory tests can be useful in diagnosis of patients with neuromuscular weakness. Diseases that damage striated muscle fibers result in leakage of intracellular enzymes into the blood, where they become measurable by serum tests. These include creatine kinase [2, 3], myoglobin, aldolase, transaminases, and lactic acid dehydrogenase [4, 5]. The presence of myoglobin in urinalysis can be a useful marker of muscle diseases. Myoglobin is an iron-protein compound present in the sarcoplasm of striated skeletal and cardiac muscle fibers, containing a red pigment that gives muscle its characteristic color. Muscle destruction regardless of cause therefore releases myoglobin, which due to its small size is filtered freely through the renal glomeruli and appears in the urine, a phenomenon referred to as "myoglobinuria" [5]. Specific autoantibody tests such as anti-acetylcholine receptor antibodies (AChR-Ab), muscle-specific tyrosine kinase (MuSK-Ab), and voltage-gated potassium channel antibodies (VGKC-Ab) can help identify specific NMJ diseases. Moreover, detection of antibodies against extractable nuclear antigens such as anti-Ro/SSA, anti-La/SSB, anti-Sm, and anti-RNP and myositis-associated antigens such as anti-histidyl-t-RNA synthase (anti-Jo-1) can be suggestive of an inflammatory myopathy or associated connective tissue disease [6].

\subsubsection{Electrodiagnostic Testing}

Electrophysiologic studies such as nerve conduction studies (NCS) and electromyography (EMG) are useful when the location of the lesion responsible for weakness is suspected to be in 
the PNS, i.e., spinal nerves, NMJ, or muscle. Our goal in the next few paragraphs is to discuss the importance of these studies in the diagnosis of neuromuscular disease. Details of NCS and EMG interpretation are beyond the scope of this chapter.

Most often, NCS, which are obtained using surface electrodes, is followed by EMG. NCS includes studies of compound muscle action potentials (CMAPs) and sensory nerve action potentials (SNAPs), which collectively provide valuable information about the integrity of motor and sensory nerve fibers. Analysis of amplitudes and latencies of CMAPs and SNAPs allows localization of the problem to axon, myelin sheath, nerve root, or branches.

EMG involves insertion of a needle with a recording electrode into muscle and observing its electrical waveforms at rest and with activation. Analysis of the waveforms allows evaluation of the integrity and function of motor units which comprises anterior horn cells, nerve axon, terminal nerve branches, and muscle fibers innervated by these branches. Collectively, NCS and EMG can be useful to localize peripheral weakness, identify characteristic features that narrow differential diagnoses, and identify affected muscles for biopsy [7]. They can differentiate between neuropathic and myopathic disease (Fig. 5.1) as well as identify defects in presynaptic or postsynaptic transmission, but the yield of these studies is higher when the electromyographer has an idea of clinically weak muscles and an idea of the differential diagnoses based on history and examination [8]. When clinically appropriate, certain specialized nerve conduction techniques can be employed, such as repetitive nerve stimulation and single fiber EMG for suspected disorders of neuromuscular transmission [6] as well as short or long exercise testing for suspected non-dystrophic myotonias and periodic paralysis [9].

The first step in performing an EMG is to assess for the presence of abnormal spontaneous activity while the muscle is at rest. Following electrode insertion into normal muscle at rest, a brief burst of electrical activity typically occurs lasting $300 \mathrm{~ms}$ or less, followed by electrical silence [10]. Neuropathic disorders exhibit abnormal spontaneous activity at rest, including fibrillations and positive sharp waves, which arise from spontaneous firing of individual muscle after denervation. In general, muscle is electrically silent in myopathies, but it is important to note that spontaneous activity may occur in certain myopathies due to irritability of the muscle membranes. This occurs particularly in inflammatory, necrotic, or certain congenital myopathies as well as muscular dystrophies [11].

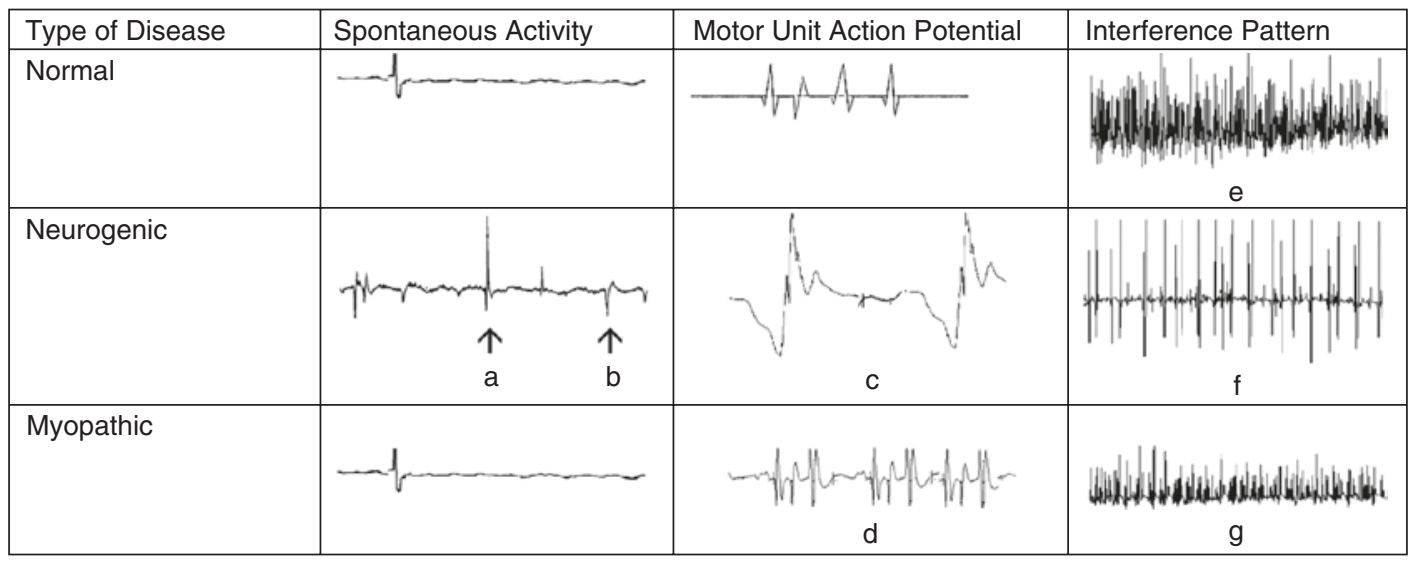

Fig. 5.1 Characteristic electromyography (EMG) features in neuropathic and myopathic types of neuromuscular disease. (1) Spontaneous activity at rest: (a) fibrillations, (b) positive sharp waves. (2) Motor unit potentials: (c) giant polyphasic units and reduced recruitment, (d) small units and increased recruitment. (3) Interference pattern: (e) full, (f) reduced units, (g) reduced amplitude, normal number of units 
Following assessment of spontaneous activity at rest, muscle is activated minimally so that motor unit action potential (MUAP) morphology including amplitude, duration, and waveform can be assessed, and the muscle power is increased so that recruitment pattern can be assessed. Recruitment is the firing pattern of additional motor units and the rate at which they are activated as the patient increases power of contractions. Recruitment testing is often challenging in the intensive care unit setting (ICU) if patients lack awareness or are unable to participate in incrementally increasing their power of contractions.

In general, neuropathic disorders affect the number of voluntary motor units. When fewer voluntary motor units are available for muscle contraction, muscle recruitment is reduced, and increased effort results in rapid firing of a reduced number of motor units. Since the remaining motor units are larger than normal, the total amplitude of the MUAP and the interference pattern is increased [8]. Myopathic disorders reduce the number of functioning muscle fibers; therefore the force generated by each motor unit is reduced, resulting in MUAPs with lower amplitudes and shorter durations. To generate the same force, more motor units are recruited and motor unit firing rate increases. These differences become more apparent with increased force. Of note, these morphologic changes are not entirely specific as MUAPs with low amplitudes, and short durations can also be seen in early reinnervation after neurogenic injury. The general EMG differences between neuropathic and myopathic disorders are outlined in Fig. 5.1, but one must note there are exceptions to this simplified table. Given nuances and overlap in electrophysiologic findings, it is important to remember that electrophysiology is only part of the clinical investigation and must be interpreted in the appropriate clinical context to determine disease pattern or arrive at the correct diagnosis.

If a muscle biopsy is required for diagnosis, EMG can help to guide the biopsy site by detecting subclinical abnormalities, especially if the patient does not have any weakness on examination or only has weakness in muscles that are not typically biopsied, like the iliopsoas. It is important to only study one side of the patient if a biopsy is planned, so the biopsy can be done on the contralateral side as needle artifacts may affect muscle biopsy interpretation.

\subsubsection{Imaging Studies}

Neuroimaging such as plain radiographs, CT, or MRI can be useful in ruling out focal mass lesions such as tumors, abscesses, bulging disks, and traumatic axonal injury in the CNS or PNS accounting for neuromuscular weakness. Moreover, MRI and ultrasound are increasingly being used to detect and characterize skeletal muscle abnormalities [12]. Like EMG, skeletal muscle MRI can be used to identify targets for muscle biopsy, especially when muscles are not clinically weak, are difficult to test, or appear normal on EMG. MRI signal abnormalities lack specificity, but certain findings can be suggestive of specific conditions, for instance, edema can suggest inflammatory myopathy and fatty infiltration can suggest an underlying disease process causing chronic muscle injury. Location and appearance of muscles that are affected can also help to narrow down the differential diagnosis. For example, atrophy and edema involving the vastus lateralis and medialis with relative sparing of the rectus femoris can help to distinguish inclusion body myositis from other myopathies [13]. If a muscle biopsy is necessary for diagnosis, MRI of the muscles not only helps to identify the optimal site for biopsy but has been shown to reduce overall cost [14]. A modality gaining popularity is ultrasound, as it is noninvasive and inexpensive and provides an assessment of muscle thickness, abnormal movements (e.g., fasciculations), and infiltration by fibrotic tissue or fat, but it lacks the imaging detail offered by MRI.

\subsubsection{Muscle Biopsy}

Diagnosis of a myopathy can be made using patient history and physical examination in conjunction with laboratory tests, electrophysiology, imaging, and sometimes genetic testing. In challenging cases, a muscle biopsy is required to make or confirm a diagnosis and remains the gold standard. 
In patients with proximal muscle weakness, elevated $\mathrm{CK}$, a myopathic EMG, or abnormal skeletal muscle MRI without a definite diagnosis, a muscle biopsy is strongly considered as it has a highly positive diagnostic outcome. In the absence of these findings, patients should be counseled that the yield of a muscle biopsy is low [15].

Common proximal biopsy sites include deltoid, biceps brachii, triceps brachii, and quadriceps, and common distal biopsy sites include forearm extensors, gastrocnemius, and tibialis anterior, but other muscles are considered based on the clinical scenarios. There are two approaches in performing a muscle biopsy, the open and percutaneous approach, and each approach has its own advantages and disadvantages. Open biopsy allows larger sampling offering the opportunity for more extensive testing due to a larger sample size, but by its open nature is more invasive. The percutaneous approach is less invasive as it involves the use of a needle with a hollow core to obtain multiple small samples; however, the samples are smaller and testing is often limited. Muscle biopsy is done on muscles that have mild to moderate weakness; muscles that are severely weak are usually of a low diagnostic yield, as they often show endstage pathologic changes that cannot distinguish between various myopathies or even severe neurogenic atrophy.

Once the biopsied tissue reaches the pathology laboratory, pieces are flash frozen in isopentane and cooled in liquid nitrogen. Various stains are then performed on these frozen sections and used for routine histochemistries, Western blots to evaluate specific proteins, enzymology to evaluate lipid or glycogen storage diseases, and genetic testing for diseases such as mitochondrial diseases. Some of the stains include hematoxylin and eosin (H\&E) staining that displays muscle architecture, a modified Gomori trichrome stain for examining structural changes, ATPase staining for visualization of fiber type-specific atrophy, and periodic acid-Schiff staining for evaluation of glycogen content. In certain cases, such as to confirm inclusion body myositis, additional specimens are placed in formalin and glutaraldehyde solution and further analyzed under electron microscopy [16].

\subsubsection{Genetic Testing}

Specific defects can be identified by Western blot or molecular analysis of mutations. Genetic testing is becoming increasingly useful in confirmation and categorization of patients with heritable diseases such as mitochondrial myopathies and muscular dystrophies [17]. When genetic defects are strongly suspected, it is advisable to obtain genetic testing prior to performing a muscle biopsy. Western blotting is useful in differentiating an enzymatic production defect from a kinetic defect. Molecular analysis of specific mutations can confirm an enzymatic deficiency detected in biochemical assay of lymphocytes, fibroblasts, or muscle tissue [17]. If single gene sequencing has failed or it is unlikely to provide a diagnosis, large-scale molecular diagnostic testing (i.e. next-generation sequencing) can be considered, especially if it is available at a low cost.

\subsection{Neuromuscular Diseases Commonly Encountered in the ICU}

\subsubsection{Critical Illness Polyneuropathy and Myopathy}

\subsubsection{Background}

Recognition that critically ill patients frequently develop new neuromuscular dysfunction was not widespread until the final decade of the twentieth century. In 1984, a series of five patients were noticed to develop flaccid paralysis with areflexia and difficulty in weaning from the ventilator at the peak of their sepsis and multiple organ dysfunction with neurophysiology indicating development of a new axonal sensorimotor polyneuropathy [18]. In 1992, persistent paralysis was observed following long-term neuromuscular blockade but attributed to higher plasma levels of vecuronium metabolites and impaired renal function [19]. Another study reported severe weakness in two critically ill asthmatic patients treated with high-dose steroids and nondepolarizing blocking agents, in whom muscle biopsy demonstrated depletion of thick myosin filaments; they called this acute quadriplegic 
myopathy and reported their clinical course over several months [20]. Many of these reports did not include detailed neurologic examination or electrophysiological testing, limiting definitive diagnosis at the time. In 1994, in 23 out of 24 critically ill comatose patients with quadriparesis or quadriplegia and diminished deep tendon reflexes (DTR), a landmark study demonstrated evidence of neuropathy, myopathy, or both based on electrophysiology [21].

As this entity was becoming more widely recognized, people began referring to this condition by several names including acute quadriplegic myopathy, acute necrotizing myopathy of intensive care, thick filament myopathy, acute corticosteroid myopathy, acute hydrocortisone myopathy, acute myopathy in severe asthma, and acute corticosteroid and pancuronium-associated myopathy, critical care myopathy, and acute myopathy of intensive care [22]. Physicians were beginning to recognize that these patients may in fact have motor recovery upon recovering from their concurrent critical illness, and the term critical illness polyneuropathy and myopathy (CINM) was coined [23].

CINM presents in critically ill patients with generalized flaccid paralysis with decreased or absent DTR and failure to wean from the ventilator. Weakness can be from an axonal polyneuropathy (critical illness polyneuropathy-CIP), a myopathy (critical illness myopathy-CIM), or both [21]. Numerous survivors of critical illness suffer from weakness and long-term disability for months to years after discharge from hospital $[23,24]$. Diagnosis of CINM is challenging in the ICU due to pre-existing disorders, other active conditions during the stay contributing to muscle weakness, and patient's clinical condition precluding careful neurologic examination. Moreover, many times in the ICU when patients are critically ill, the focus becomes patient survival, and less attention is paid to the gradually progressive neuromuscular weakness from CINM in the background. CINM is a major contributor of ICU-acquired weakness, resulting in chronic disability in survivors of critical illness, and intensivists should be familiar with its diagnosis and clinical course.

\subsubsection{Epidemiology}

A lack of consistent nomenclature, variation in patient population, diagnostic criteria used, and timing of assessment have been a barrier to determining exact incidence of CINM [23, 25]. Reported incidence of CINM is estimated to be as high as $25.3 \%$ of patients that are mechanically ventilated for 7 or more days [26], $60 \%$ of patients with acute respiratory distress syndrome (ARDS) [27], 50-84\% of mechanically ventilated ICU patients with sepsis and multiorgan failure [28, 29], and 95-100\% of mechanically ventilated patients with sepsis and coma $[21,30]$.

\subsubsection{Risk Factors and Pathophysiology}

Systemic inflammatory response syndrome (SIRS), sepsis, sepsis with mechanical ventilation [26], and multiorgan failure appear to consistently be risk factors for CINM [28, 29, 31-33]. Diaphragmatic weakness, a prominent feature in CINM, has been observed to rapidly develop during mechanical ventilation and is significantly related to the duration of ventilator support [34]. A high catabolic state from systemic inflammation likely contributes to early diaphragmatic weakness in these patients. The strong occurrence of CINM with sepsis and MODS suggests that there are likely shared metabolic, cellular, and microcirculatory pathophysiological mechanisms. Sepsis results in ischemic hypoxia due to widespread impairment in microcirculation [35] as well as impaired mitochondrial function resulting in reduced adenosine triphosphate (ATP) production [36, 37]. Excitable tissue like peripheral nerves and muscle are probably damaged as a result of this microcirculatory failure, resulting in distal axonopathy [38]. In patients with CIP, the expression of E-selectin, a marker of endothelial activation, is increased in vascular endothelium [39]. This could result in increased leukocyte adhesion, local cytokine production, increased microvascular permeability, and endoneurial edema, which could be further worsened by hypoalbuminemia and hyperglycemia. In addition, decreased nutrient supply, increased demand, and decreased clearance of toxic substances and tissue edema can ultimately terminate 
in bioenergetic failure, neuronal injury, and axonal degeneration. Pathologically in CIN, there is axonal degeneration without inflammation.

Other risk factors for CINM include prolonged ICU stay [40-42], hyperglycemia [40], hypoalbuminemia [40], duration of vasopressor and catecholamine support [41], hyperosmolarity [42], parenteral nutrition [42], and neurologic failure $[25,42]$. This data is conflicting for nondepolarizing neuromuscular blockers (NMB) and steroids. High corticosteroid levels are thought to increase expression of ubiquitin, leading to increased proteolytic activity [43]. NMBs have been identified as risk factor [25, 42], but a large study showed that early administration of cisatracurium improved 90-day ARDS patient survival and increased the time off the ventilator without increasing muscle weakness [44]. This finding could be explained by either, the intermediate-effect duration of cisatracurium, or the fact that cisatracurium was only used for 48 hours in the study. It is postulated that nondepolarizing NMBs cause muscle weakness by upregulation of acetylcholine receptors with fetal-type receptors that are less responsive to acetylcholine, presynaptic inhibition of exocytosis of acetylcholine, and potentiation of muscle damage by corticosteroids [45].

Immobility and muscle inactivity acts as a potent stimulus of the ubiquitin-proteasome pathway of proteolysis in skeletal muscle, increasing muscle wasting [46] during critical illness. Denervation from coexisting CINM may make the muscle more susceptible. Muscle biopsy in CIM shows loss of thick myosin (thick) filaments, fiber-type atrophy, and/or necrosis [20, 47]. Whether the different pathologies represent a spectrum of severity of the same disease or completely different entities is unsettled.

\subsubsection{Clinical Presentation}

CINM is most often diagnosed when sedation is weaned or sepsis and encephalopathy improve in a mechanically ventilated patient, revealing a profound generalized weakness that cannot be explained by coexisting neurologic disease, metabolic disturbances, increased metabolic demand, nutritional disorders, profound anemia, or delirium. In a patient who is alert, muscle strength can be tested in functional limb muscle groups with the Medical Research Council (MRC) scale. Neurologic exam reveals normal cranial nerves with generalized flaccid quadriparesis or quadriplegia, often worse distally, usually in conjunction with failure to wean from the ventilator. Hyporeflexia, areflexia, and muscle atrophy can be seen in CIP, though reflexes are usually preserved or hypoactive in CIM. Distal sensory loss to pain, temperature, and vibration may be observed in alert patients [48] but is not always reported or amenable to testing [49]. When noxious stimulus is applied, one may observe facial grimacing but reduced or absent movement of the limbs. Sensation is normal in CIM if neuropathy does not coexist, but testing is often limited by coexisting encephalopathy [46]. Electrodiagnostic studies can therefore be key to diagnosis, though a high index of clinical suspicion is prerequisite.

\subsubsection{Diagnosis}

CIP is a distal sensory-motor axonal polyneuropathy affecting limb and respiratory muscles, sparing cranial nerves [21]. Muscle weakness in the limbs is symmetric and worse distally than proximally. Diagnostic criteria exist for CIP and CIM and are outlined in Table 5.2. A high index of clinical suspicion must exist if patient develops new neuromuscular weakness after survival of critical illness, SIRS, sepsis, and/or MODS. Alternative causes for neuromuscular weakness in the ICU must be considered and, if possible, excluded based on history and examination (refer to Table 5.1). Laboratory studies can help to exclude mimics, including muscle enzymes such as CK. Depending on clinical suspicion, imaging of the brain and spinal cord may be appropriate to exclude other disease of the CNS and PNS such as tumors, infections, and disk diseases. Cerebrospinal fluid findings are not often reported in CIP. Lumbar puncture is indicated principally if there is clinical suspicion of CNS or PNS infection.

Electrophysiologic testing and muscle biopsy can help confirm CIM or CIP and help to distinguish them from each other as well as other 
Table 5.2 Diagnostic criteria for critical illness polyneuropathy (CIP) and critical illness myopathy (CIM)

\begin{tabular}{l|l}
\hline CIP & CIM $^{\mathrm{b}}$ \\
\hline $\begin{array}{l}\text { The patient is critically } \\
\text { ill (with SIRS, sepsis, } \\
\text { and MODS) }\end{array}$ & $\begin{array}{l}\text { SNAP amplitudes }>80 \% \text { of } \\
\text { the lower limit of normal }\end{array}$ \\
\hline $\begin{array}{l}\text { Difficulty weaning } \\
\text { patient from ventilator } \\
\text { after nonneuromuscular } \\
\text { causes such as heart } \\
\text { and lung disease have } \\
\text { been excluded }\end{array}$ & $\begin{array}{l}\text { Needle EMG with } \\
\text { short-duration, low- } \\
\text { amplitude MUPs with early } \\
\text { or normal full recruitment, } \\
\text { with or without fibrillation } \\
\text { potentials }\end{array}$ \\
\hline $\begin{array}{l}\text { Possible limb weakness } \\
\text { Absence of a decremental } \\
\text { response on repetitive nerve } \\
\text { evidence of axonal } \\
\text { motor and sensory } \\
\text { polyneuropathy }\end{array}$ & $\begin{array}{l}\text { Muscle histopathologic } \\
\text { findings of myopathy with } \\
\text { myosin loss }\end{array}$ \\
\hline $\begin{array}{l}\text { CMAP amplitudes }<80 \% \text { of } \\
\text { the lower limit of normal in } \\
\text { two or more nerves without } \\
\text { conduction block }\end{array}$ \\
\hline $\begin{array}{l}\text { Elevated serum creatine } \\
\text { kinase (CK) }\end{array}$ \\
$\begin{array}{l}\text { Demonstration of muscle } \\
\text { inexcitability }\end{array}$ \\
\hline
\end{tabular}

${ }^{a}$ These diagnostic criteria are now well established, originally created by Bolton [38]. Other acute axonal polyneuropathies should be excluded

${ }^{b}$ For a definite diagnosis of CIM, patients should have all of the first five features

disorders of nerve, NMJ, and muscle. Nerve conduction studies may be difficult to perform and interpret due to multiple artifacts, extremities may be cool and difficult to warm, and they may be confounded by tissue edema. EMG may not be useful when patients are not awake or unable to contract muscles to command. Nonetheless, they are usually diagnostic [38].

Nerve conduction studies in CIP show decreased CMAP and SNAP amplitudes with normal or mildly reduced conduction velocities and normal CMAP durations. The abnormalities are usually length-dependent. NCS findings are generally present by day 14 of critical illness, but low amplitudes may be noted within a week and as early as $72 \mathrm{~h}$ after onset of sepsis [49]. Needle EMG studies in CIP may show fibrillation potentials and positive sharp waves in a multifocal pattern generally after 2 weeks but have been reported as early as 7 days after initiation of mechanical ventilation [33]. Muscle biopsy in CIP shows evidence of acute denervation of muscle with atrophy of both type 1 and type 2 fibers and grouped atrophy as recovery occurs. Nerve biopsy is rarely indicated but if done will show signs of axonal neuropathy [21].

In patients with CIM, the CMAP is also of low amplitude, but unlike CIP, CMAP duration is prolonged due to slowing of muscle fiber conduction velocity and reduced excitability of the sarcolemma membrane. These changes occur within 2 weeks of critical illness [50]. SNAPs are normal in CIM, unless there is coexistent CIP. Voluntary muscle activity may be difficult to study in poorly cooperative patients, but when performed, reduced recruitment is seen in CIP, and small polyphasic motor unit potentials are seen in myopathy, helping to differentiate CIP from CIM.

Muscle biopsy in CIM shows selective loss of myosin and varying degrees of muscle necrosis directly proportional to disease severity $[21,51]$. During recovery in CIM, electrophysiological studies show a rise in CMAP amplitude, normal CMAP duration as well as loss of fibrillation potentials, and/or positive sharp waves from muscle. There is a wide variance in electrophysiology based on severity of disease. In severe CIPM where CIP and CIM coexist, CMAP and SNAP amplitudes are considerably reduced, and muscle biopsy shows substantial, generalized necrosis. Although muscle and nerve biopsies may be diagnostic, they often only change management if they demonstrate an alternative diagnosis that is treatable, for instance, an inflammatory myopathy or demyelinating or vasculitic neuropathy. If the clinical index of suspicion is high for CINM, a biopsy is likely not warranted.

Additional electrodiagnostic studies that may be useful in diagnosis include phrenic nerve stimulation and diaphragmatic EMG, but many electromyographers do not have necessary experience with them to be confident in their performance or interpretation. Phrenic nerve stimulation in the neck with surface recording over the diaphragm shows a normal response in patients with pulmonary causes of respiratory failure but a low amplitude or absent response in patients with neuromuscular respiratory weakness. Needle EMG of the diaphragm can be done in patients without severe chronic obstructive pulmonary disease (COPD), ileus, or coagulopathy and may 
diagnose denervation or myopathy affecting the diaphragm [52].

\subsubsection{Management}

Since no specific therapy that decreases incidence or severity of CINM has been found, management has been largely supportive [53]. Treatment of hyperglycemia, early physical rehabilitation, and minimization of sedation have been demonstrated to improve short-term and long-term functional outcomes, increase ventilator-free days, and reduce delirium [54]. Of the factors that may contribute to the development of CINM, many-like the response to sepsis and the incidence of multiorgan failure-are related to the severity of the presenting critical illness and are not easily modifiable. It remains to be demonstrated whether the reduced use of corticosteroids or neuromuscular blocking agents will result in a decreased incidence of CIM. Intensive insulin therapy to maintain strict normoglycemia was shown to reduce the incidence of critical illness polyneuropathy by $49 \%$ in a single-center study [41], but this practice has been shown to increase mortality in patients who are critically ill [55]. Therefore, control of sustained hyperglycemia is reasonable, but aggressive insulin protocols and any hypoglycemic episodes should be avoided [55]. Patients with neuropathy are more prone to pressure palsies, and, thus, great care must be taken in positioning and support to avoid this complication. Neurotoxic medications should also be avoided. In patients that are unable to participate in active strengthening exercises, early physical therapy should be directed toward prevention of muscle atrophy and contractures, as studies have shown that repeated daily passive mobilization prevents muscle atrophy observed by serial muscle biopsies [56]. Early rehabilitation in the ICU requires a cultural shift and a strong commitment from a multidisciplinary team but improves functional outcome [54].

\subsubsection{Prognosis}

There is no comprehensive study on prognosis in CINM. Mortality is high early in the course and is related to the underlying critical illness [34]. Patients with CINM who survive the acute illness and who begin to recover strength are likely to continue to recover over the weeks to months that follow, and it is sufficient to monitor improvement with clinical assessment of strength. Patients with severe CINM have slow and often incomplete recovery, and nearly a third of patients with CINM do not recover spontaneous ventilation or independent walking [25]. Patients with CINM who fail to show clinical improvement in strength can be evaluated with repeat electrophysiologic testing. Patients with evidence of severe axonal loss, with small or absent responses to nerve stimulation and abundant positive waves and fibrillations on needle EMG, will recover slowly if at all and are likely to have residual deficits-sometimes severe [40, 48]. The task of regenerating severely damaged axons appears more daunting than regenerating muscle [21], but recent evidence suggests CIM to predict a better functional recovery at both time of discharge and 1 year follow-up [57].

\subsubsection{Future Directions}

A good understanding of the pathophysiology of CINM is necessary to be able to develop goaldirected interventions, and recent discoveries make this a realistic possibility in the future. Skeletal muscle regeneration is reliant on stem (satellite); this is supported by human evidence that shows that decreased satellite cell content impedes muscle growth and results in protracted muscle wasting [58]. Emerging evidence shows that impairment of satellite cells leads to inefficient muscle regeneration and engrafting mesenchymal stem cells restores metabolic and mitochondrial function of satellite cells, improving muscle strength in a mice model [59]. An experimental ICU porcine model study that reproduced ICU conditions such as sedation, long-term mechanical ventilation, and muscle unloading showed that mechanical silencing is a dominant factor triggering the preferential loss of myosin and muscle atrophy in a period of within $6 \mathrm{~h}$ to 14 days [60]. In a later study, the same authors attempted to identify gene signatures and molecular pathways that regulate mechanical activation of skeletal muscle affected in the ICU; they found that mechanical silencing triggers genes that regulate mitochondrial dynamics and mitophagy as well as ubiquitin 
ligases Fbxo31 and SMART, which are all reversed by passive mechanical loading [61]. This may explain the beneficial effects of early mobilization and physical therapy in CINM. CINM continues to be an area of active research and discovery, and it is our hope that we will have better understanding and be able to tailor therapy accordingly in the future.

\subsubsection{Myasthenia Gravis}

\subsubsection{Background}

Myasthenia gravis (MG) is the most common autoimmune disorder of the NMJ. It is caused by autoantibodies against receptors located in postsynaptic cleft, which affects neuromuscular synaptic transmission [62]. The first documented case of MG in literature was in a Native American, Chief Opechankanough, who died in 1664 [63, 64]. However, the autoimmune nature of the disease was established in 1973 when first antibodies to acetylcholine receptor (AchR) were identified [65]. Since then, there has been major progress in understanding the pathophysiology of the disease and introduction of multiple treatment options which has led to improvement in outcome and mortality of the patients with MG.

\subsubsection{Epidemiology}

The incidence of MG incidence varies from 0.25 to 2.0 per one million people with prevalence of 15-179 per one million people worldwide of which $10 \%$ are in pediatric population $[62,66$, 67]. It has a bimodal distribution with early-onset disease seen in second and third decades of life with a female predominance and late-onset disease which is seen in sixth to eighth decade of life and has a male predominance [68].

\subsubsection{Pathophysiology}

The NMJ consists of the terminal branch of the motor axon, synaptic cleft and postsynaptic muscle fibers. When a wave of depolarization reaches the terminal branch of an axon at NMJ, it causes calcium channels located in the membrane of the axon to open and an influx of calcium follows. This increase in intracellular calcium mobilizes the vesicles containing acetylcholine to move closer to the membrane and subsequently release acetylcholine into synaptic cleft. When the released acetylcholine binds to its receptor on muscle fibers, it causes a small and focal depolarization of the membrane called miniature endplate potential (MEPP). The summation of these MEPPs eventually exceeds the depolarization threshold of the muscle fiber, resulting in muscle contraction.

MG is an autoimmune disorder with autoantibodies against receptors at NMJ. Three major receptors have been identified that are affected by autoantibodies resulting in muscle weakness as the main clinical symptom of MG. In this section, we will review these three receptors, the autoantibodies and their role in NMJ and muscular weakness.

Acetylcholine receptor is a nicotinic receptor located on the postsynaptic muscle fiber membrane of skeletal muscles. This receptor is made of five homologous subunits that are arranged in a circular fashion around a central pore [69]. The $\alpha 1$ subunit which is called the main immunogenic region (MIR) is the main target for AchR autoantibodies [70]. They are detected in $85-90 \%$ of generalized MG and 40-70\% of ocular MG patients [71]. AchR antibodies are of IgG1 and IgG3 subclass of antibodies [72] that disrupt the process of neuromuscular transport/contraction by three primary mechanisms: (1) attach to MIR and directly inhibit AchR; (2) activate complement, causing lysis of the postsynaptic membrane, changing synaptic configuration and subsequently destruction of the acetylcholine receptors located in this area [73, 74]; and (3) induction of AchR endocytosis and lysosomal destruction by autoantibody-mediated crosslinking of AchR, which decreases the number of available functioning Ach receptors at the surface of muscle fibers [75, 76].

Muscle-specific tyrosine kinase receptor (MuSK) was first identified in 2001 [77]. This receptor has an important role in clustering of postsynaptic AchR in NMJ through agrin, which results in spatial arrangement of AchR required for proper function of NMJ [78]. Autoantibodies against MuSK are of the IgG4 subclass and 
directly inhibit MuSK receptors at NMJ with no activation of complement as part of autoimmune process which explains no loss of AchR or complement deposition as seen in AchR antibodypositive patients [79]. Of note, MuSK-MG has a marked female predominance and $40 \%$ of patients with generalized, acetylcholine receptor antibody (AChR-Ab)-negative MG had MuSK-MG [62]. Lipoprotein-related protein 4 (LRP4), identified in 2011, is a membrane protein, which is also responsible for clustering of AchR though agrinLRP4 interaction. LRP4 autoantibodies are positive in $9 \%$ of patients who are negative for both AchR and MuSK antibodies [80].

\subsubsection{Clinical Presentation}

The hallmark of MG is muscle weakness that varies throughout the day and that worsens with exercise and improves with rest. The majority of patients (about $80 \%$ ) have generalized MG, but in $20 \%$ of cases, the muscle weakness can be limited to ocular muscles such as the levator palpebrae, and this subtype is known as ocular MG [81]. This muscle weakness is asymmetric and results in double vision and ptosis, which can be transient or fluctuate throughout the day. Nearly $50-80 \%$ of patients who primarily present with symptoms limited to ocular muscles develop systemic MG within 2 years of initial presentation (secondary generalized MG); therefore, it is reasonable to postpone the diagnosis of ocular MG for 2 years after initial presentation given the high rate of developing SGMG [82-84].

Pattern of muscular weakness in patients who are found to be positive for MuSK antibody is usually different from what is seen in patients who are AchR positive. In comparison with AchRpositive MG patients, there is a female predominance in MuSK-positive myasthenia patients with a younger age of onset, mostly less than 60 years of age [85]. These patients most often present with bulbar weakness associated with tongue and facial atrophy, neck and shoulder weakness, and respiratory failure secondary to respiratory muscle weakness without ocular involvement. However, they can also present with similar symptoms indiscernible to AchR-positive patients [72]. Myasthenic crisis is defined as respiratory failure secondary to muscular weakness. Around $20 \%$ of patients with myasthenia will experience one episode of crisis throughout their disease course, and it may be their initial presenting symptom that leads to the diagnosis of MG $[86,87]$.

\subsubsection{Diagnosis}

Diagnosis of MG is made based on a combination of history, typical physical examination findings, serologic testing and electrophysiologic studies. Bedside testing such as the edrophonium ("Tensilon") test have high sensitivity but have fallen out of favor due to low specificity and high interpreter variability.

Nerve Conduction Studies (NCS) and Electromyography (EMG)

Sensory and NCS studies in MG patient are generally normal but may show low CMAP amplitudes in muscles that demonstrate severe weakness. Slow repetitive nerve stimulation (RNS) with $2-3 \mathrm{~Hz}$ is a specialized test that has over $80 \%$ sensitivity in generalized MG [88]. RNS evaluates the safety factor which is the number of acetylcholine receptors on the post synaptic junction that is required to form an adequate endplate potential causing membrane depolarization and muscle contraction. In MG, there are not enough acetylcholine receptors available, and the safety factor is decreased. A decrement of more than $10 \%$ in CMAP amplitude is seen after slow repetitive stimulation and is more pronounced between the first and second stimuli. RNS usually is done on distal muscles first, but if the results are negative, proximal muscles like trapezius or nasalis muscles and in cases of ocular myasthenia, the orbicularis oculi muscle should be tested. It is important to remember that RNS should not be performed near a pacemaker or indwelling central venous catheter, and all anticholinergic medications should to be discontinued for $12 \mathrm{~h}$ prior to edrophonium testing [8].

On EMG of MG patients, the most significant finding is moment-to-moment variation in the amplitude of MUAP, which requires studying one single MUAP over a period of time. The changes in the MUAP amplitude have the same meaning as the decrements in RNS [8]. Single fiber EMG is the gold standard for 
diagnosis of NMJ disorders which shows increased jitter in MUAPs. However, since it is a technically difficult procedure, it is reserved for when there is high suspicion for the disease and RNS is negative [8].

\subsubsection{Trial of Cholinesterase Inhibitor}

The administration of edrophonium, a cholinesterase inhibitor is described here for historical reasons but the drug is no longer available in the United States and many other countries. Edrophonium is a short-acting (5-10 $\mathrm{min})$ cholinesterase inhibitor with fast onset (30-45 seconds). A positive test for MG is characterized by by rapid improvement of symptoms upon administration. This test should only be used in patients with ocular myasthenia or patient with general myasthenia who have a pronounced ptosis or ophthalmoparesis in whom the improvement of symptoms can easily be evaluated. It has a sensitivity of $80-90 \%$ but is not specific to MG.

The test begins with the administration of $2 \mathrm{mg}$ of edrophonium intravenously followed by $2 \mathrm{mg}$ every $60 \mathrm{~s}$, up to total of $10 \mathrm{mg}$. This slow incremental dosing will ensure that patients get enough medication to improve their symptoms while avoiding cholinergic side effects as much as possible. However, patients can still develop cholinergic side effects including bradycardia, bronchospasm, gastrointestinal cramping, and increased salivation. Atropine should be available for immediate intravenous administration if side effects occur. This test should be avoided in elderly patient and those with cardiac abnormalities such as bradycardia or conduction blocks or bronchospasm [89].

\subsubsection{Ice Pack Test}

The ice pack test is performed in patients whom edrophonium testing is contraindicated. It has $80 \%$ sensitivity and can be done at bedside or in the office. It is only useful in patients with ptosis and cannot be used in patients with ophthalmoparesis since those muscles are located deeper and cannot be sufficiently cooled by ice packs. The test is performed by placing the ice pack on the eye with ptosis for 2 min followed by immediate evaluation of the degree of improvement in ptosis $[90,91]$.

\subsubsection{Management}

Significant decrease in MG mortality in recent decades is likely attributable to advancements in both ICU care as well as in immunomodulatory therapies such as intravenous immunoglobulin (IVIG) and plasma exchange (PLEX). MG treatment options can be divided in three categories: symptomatic therapy, chronic immunosuppressive therapy, and rapid immunomodulating therapy.

The first category of treatment options is symptomatic therapy. The most commonly used medication used for symptomatic relief is pyridostigmine, which is an acetylcholinesterase inhibitor. Cholinergic side effects of this medication are the main reason for noncompliance in patients. Diarrhea can be a major issue in elderly patients who have underlying problems with sphincter control. Loperamide can help alleviate diarrhea without affecting NMJ [92]. Patients with MuSK autoantibody do not respond as well to usual doses of pyridostigmine and require higher doses, which increases the systemic side effects of the medication [93]. However the side effects of the medication are seen at doses higher than $300 \mathrm{mg} /$ day. If pyridostigmine bromide cannot be tolerated secondary to GI side effects, ambenonium chloride can be used as a replacement [94].

The second category of treatment options is chronic immunosuppressive therapy. There are multiple medications in this category, but the two mostly used agents as first-line therapy are corticosteroids and azathioprine, a steroid-sparing immunosuppressant. Second-line agents are reserved for patients with poor response to initial therapy. These include, cyclosporine A, methotrexate, mycophenolate mofetil, and tacrolimus.

Corticosteroids can improve symptoms in $70-80 \%$ of patients within $4-8$ weeks, and there are different regimen to choose from, including intravenous methylprednisolone (MP) and oral prednisone:

- Intravenous MP: Dosing can vary between $500 \mathrm{mg}$ and $2 \mathrm{~g}$ daily for 3-5 days followed by an oral prednisone taper $[95,96]$. Administration of such high-dose steroids can lead to a transient worsening of myasthenia symptoms and in some cases a myasthenia crisis. 
For this reason, this treatment is reserved for patients with myasthenia crisis and in combination with other acute phase treatments, like IVIG and plasmapheresis. It is important to remember that steroids can cause peptic ulcers and osteoporosis among other side effects like HTN and psychosis, which makes it crucial for all these patients to be treated with calcium (1000-1500 mg/day) and vitamin D (400$800 \mathrm{IE} /$ day) as well as proton-pump inhibitors (PPI) or H2 blockers for prevention of osteoporosis and peptic ulcers, respectively [94].

- Oral prednisone: 10-20 mg as initial dose, with weekly increase by $5 \mathrm{mg} /$ day until patient's symptoms improve or stabilize [97]. The disadvantage of this regimen is the slow speed of recovery compare to higher doses of steroids; however, it prevents transient worsening of muscle weakness and clinical symptoms upon initiation of therapy [94].

- Oral prednisone: Initial dose of 1-1.5 mg/day/ $\mathrm{kg}$ bodyweight in conjunction with a steroidsparing immunosuppressant. Dose can later be decreased by $5 \mathrm{mg} /$ day every 4 weeks when clinical symptoms improve with goal to completely discontinue steroids. This regimen can result in rapid recovery and resolution of symptoms; however, about $10 \%$ of patients will experience transient decline and worsening of muscle weakness with initiation of therapy $[98,99]$.

Steroid-sparing immunosuppressants include:

- Azathioprine (AZA): is the first-line agent in this group for treatment of MG [100]. AZA derivative, 6-mercaptopurine, acts by prohibiting activation and proliferation of $B$ cells and $\mathrm{T}$ cells in the body leading to its immunosuppressant effect. The starting dose is $2-3 \mathrm{mg} /$ day $/ \mathrm{kg}$, which can later be decreased to as low as $1 \mathrm{mg} /$ day $/ \mathrm{kg}$ bodyweight if the patient's clinical symptoms have been stabilized or remission has been achieved. Treatment effects of AZA will be seen months after initiation of therapy [101]. AZA in combination with steroids can lead to lower doses of steroids required, more effective treatments, and less side effects [102] although in 10-20\% of patients who are resistant to therapy, higher doses of steroids may be required despite use of AZA. AZA treatment should not be abruptly discontinued since it can cause recurrence of MG symptoms and at times MG crisis [103, 104]. Side effects seen in patients treated with AZA are increased MCV, reversible lymphopenia, nausea, vomiting, and diarrhea [105]. At times, AZA dose can be decreased by $25 \%$ with addition allopurinol that prevents AZA metabolization and hence decreases the myelotoxic side effects of AZA [94].

- Ciclosporin a (CSA): has been proven to be effective in treatment of MG in one placebocontrolled trial [106]. The initial dose is $3-4 \mathrm{mg} /$ day/kg in two single doses and in conjunction with steroids. This dose can later be reduced to $2-2.5 \mathrm{mg} / \mathrm{day} / \mathrm{kg}$ as the patient's disease stabilizes. Ciclosporin compared to AZA has a more rapid clinical affect, usually within 4-6 weeks, but it also has worst side effect profile with myelosuppression, opportunistic infections, and nephrotoxicity leading to hyperkalemia. It can decrease seizure threshold and cause reversible posterior leukoencephalopathy syndrome [94].

- Methotrexate (MTX): causes its immunosuppressant effects by lowering the proliferation of lymphocytes through preventing the production of DNA, RNA, and proteins. Based on a recent clinical trial published in 2011 done by Heckmann J et al., MTX had equivalent effect in steroid-sparing properties when compared to AZA in a span of 2 years [107]. Hence MTX at doses 7.5-25 mg/week can be used as a secondline agent for MG treatment. Patient should also be prescribed folic acid supplements in conjunction with MTX [108]. Hepatotoxicity, anemia, leukopenia, nausea, vomiting, abdominal pain, renal failure, and acute pneumonitis are some of the side effects of MTX.

- Mycophenolate mofetil (MMF): has been shown to improve clinical symptoms of MG when used in doses of 1500-2000 mg/day [109-111]. Although there were two studies that did not show any superiority to prednisone [112] and no steroid-sparing benefit in 9 months 
[113], it was thought that a 9-month follow-up period was not long enough to evaluate the efficacy of MMF since the clinical effects of the medication take a long time to be seen.

- Tacrolimus (TCM): like ciclosporin A, works by inhibiting calcineurin and lymphocyte activation. However, it is $10-100$ times stronger than CSA and causes the same side effects. It has been shown that TCM in doses of 3-5 mg/ day can be beneficial for treatment of patients with MG [114-117]. In one cohort study of 79 patients with MG, the effects of TCM was compared to the combination of CSA and prednisone which showed symptom stabilization and decreasing levels of AchR antibodies $[118,119]$. Drug's blood level should be monitored for nephrotoxicity and neurotoxicity side effects.

- Rituximab: is an antiCD20 monoclonal antibody, which causes immunosuppression by decreasing the number B lymphocytes in circulation. A recent metanalysis, published in 2015, looking at 15 studies and 168 patients, showed a response rate of $83.9 \%$ to rituximab. The patients in these studies were either positive for AchR antibodies or MuSK antibodies or were negative for both of these antibodies. The rituximab dose used in these 15 studies was also different. This metanalysis showed that patients with positive MuSK antibodies had a better response to rituximab treatment compared to the rest of the patients [120]. This was thought to be secondary to the fact that IgG4 antibodies in MuSKpositive patient were made by CD20+ plasma cells in blood that are more affected by rituximab [121, 122].

- Cyclophosphamide (CPP): can be used in cases of severe MG that has not responded to standard treatments or those who require frequent plasmapheresis or immunoadsorption [123]. It can be administered as pulse therapy with $500 \mathrm{mg} / \mathrm{m}^{2}$ body surface area every 4 weeks [124] or immunoablative therapy with $50 \mathrm{mg} /$ day $/ \mathrm{kg}$ for 4 consecutive days $[123,125]$. The latter dose should be followed by GCSF or stem cell transplantation $[125,126]$.
The third category of treatment options, rapid immunotherapy such as intravenous immunoglobulin (IVIG) and plasmapheresis/plasma exchange (PLEX), is the main category of therapies to treat myasthenic crises. They are rapid in onset but short-acting, only lasting up to a few weeks. They are also used as bridge therapies to slower-acting immunosuppressive therapies, preoperative optimization prior to thymectomies, treatment in patients who have contraindications for other immunosuppressant therapies or refractory MG patients who have failed standard immunosuppressive therapies. IVIG is pooled immunoglobulin from donors. Mechanism for IVIG in MG is unclear. IVIG can be administered as $0.4 \mathrm{~g} /$ day $/ \mathrm{kg}$ in 5 days or $1 \mathrm{~g} /$ day $/ \mathrm{kg}$ in 2 days $[127,128]$. IVIG has been shown to be as effective as plasmapheresis in resolution of $\mathrm{MG}$ crisis and to decrease the time required for mechanical ventilation [129]. Side effects of IVIG include headache, hypertension, pulmonary edema, heart failure exacerbation secondary to volume overload, thrombotic and thromboembolic events like venous thrombosis, strokes and myocardial infarctions, infections, aseptic meningitis, and anaphylactic reaction especially in patients with IgA deficiency [130].

Therapeutic PLEX directly removes AChR antibodies from circulating blood and clinical improvement with plasmapheresis roughly correlates with the reduction in antibody levels. It is usually 5-8 treatments that are administered every other day [131]. With each treatment human albumin is used as a substitute, and in patients who have IgG levels of less than $150 \mathrm{mg} /$ $\mathrm{dl}$, polyvalent $\mathrm{IgG}$ is administered. It is important to remember that with plasmapheresis, coagulation factors are also depleted; therefore careful attention to signs of bleeding or use of concomitant anticoagulation drugs is prudent [132]. Studies have shown similar efficacy between plasma exchange and IVIG [133-136]. All newly diagnosed MG patients must undergo CT chest or MRI for thymoma screening.

Ten to fifteen percent of AchR antibodypositive patients are found to have a thymoma on CT chest [137, 138]. In this group of patients, thymectomy is indicated mainly to treat the tumor 
and prevent local invasion but also to help with myasthenia symptoms. Of patients with positive AchR antibody and no thymoma on the CT or MRI, 60-70\% have hyperplasia [137, 138]. A MG treatment trial published in 2016 randomized 126 patients with generalized MG, positive AchR antibody and disease duration of less than 5 years to thymectomy, and alternate-day prednisone alone over 3 years. Patients who underwent a thymectomy had lower quantitative MG score, lower requirements for alternate-day prednisone, lower requirements for immunosuppression, lower rates of MG exacerbation and hospitalization, and higher improvement in clinical status, indicative of beneficial clinical outcome in non-thymomatous patients who underwent thymectomy [139].

The Association of British Neurologists guidelines for the management of MG recommends thymectomy for patients less than 45 years of age and positive AchR antibodies as long as the disease is well controlled prior to operation and surgery is being done by an experienced surgeon in a facility that works closely with neurologists experienced in MG. This procedure can result in remission, prevention of generalization of ocular myasthenia, and decrease corticosteroid dose required to control the disease [140]. Thymic pathologies are rare in patients with MuSK-positive antibodies [141-144], and only a few of the patients improve clinically after thymectomies [85, 145].

\subsubsection{Guillain-Barré Syndrome (GBS)}

\subsubsection{Background}

Guillain-Barré syndrome (GBS) is an immunemediated polyradiculoneuropathy that is caused by antibodies against antigens situated on neurons which leads to destruction of myelin, axons, or both.

\subsubsection{Epidemiology}

In Europe and North America, GBS incidence is between 0.8 and 1.9 per 100,000 people per year, with a mild degree of male predominance [146]. Incidence increases with age up to 2.7 per
100,000 people per year in patients older than age 80 [147]. GBS is responsible for over 6000 hospital admission per year in the United States (US) [148]. Increased geographic incidence rates that have been published are likely related to increased rates of preceding infections in those areas [149]. Miller Fisher, a variant of GBS, made up 5\% of the cases in Western Europe with higher rates seen in Japan and Taiwan [150].

\subsubsection{Pathogenesis}

There is a proven relationship between GBS and infections by Campylobacter jejuni, Cytomegalovirus, Epstein-Barr virus, Influenza A, hepatitis $\mathrm{A} / \mathrm{B} / \mathrm{E}$, Haemophilus influenzae, Mycoplasma pneumoniae, and most recently Zika virus [151-154]. Pathogenesis is thought to be due to molecular mimicry, i.e., an immune response to a preceding infection that cross reacts with peripheral nerve components, causing the immune system to attack peripheral nerves and their spinal roots [155-157]. The nature of the microbial agent that causes the preceding infection has a role in clinical phenotype and patient's outcome [158, 159].

GBS is a heterogenous disorder with many variant forms, and each form has distinct clinical presentations, pathophysiology, electrophysiologic findings, and pathology. The three main variants are a purely demyelinating form, known as acute inflammatory demyelinating polyneuropathy (AIDP) accounting for over $90 \%$ of the cases of GBS in the United States and Europe [160]; a purely axonal form, known as acute motor axonal neuropathy (AMAN); and acute motor and sensory axonal neuropathy (AMSAN).

In AMAN, ganglioside antigens located on the axonal membrane (axolemma) are attacked by antibodies of IgG1 and IgG3 subclass, which are produced in response to an infection. The main two antigens in this category are GM1 and GD1a [161]. The binding of these antigens and antibodies activates complement with subsequent attraction of macrophages to the area, which leads to destruction of axolemma at nerve terminals and nodes of Ranvier causing conduction block in involved neurons [162]. GQ1b is another ganglioside antigen, mainly located in motor 
neurons of extraocular muscles. Antibodies to this antigen can cause one of the variants of GBS known as Miller Fisher syndrome [163, 164]. Campylobacter jejuni is the most common infection preceding GBS, found in $25-50 \%$ of patients with AMAN [154, 165].

The mechanism for AIDP is less clear, because of larger number of presumed factors (bacterial and viral infections) that stimulate the immune system and yet to be identified antibodies that attack the neuronal antigens [155]. There have been small studies that indicated immune responses from $\mathrm{B}$ cell and $\mathrm{T}$ cell to compact myelin proteins (P0, P2, and PMP22) [166].

\subsubsection{Clinical Presentation}

Hallmark of this disease is rapidly progressive, fairly symmetric, bilateral ascending weakness.

Patients often experience paresthesia in limbs distally with associated severe back pain and a tight band feeling around their torso. These symptoms usually appear 1-2 weeks after the presumed infection [167]. As the disease progresses, paresthesia spreads to all limbs, and 1-2 days later, patients develop proximal muscle weakness usually in the legs, described as difficulty standing up from a sitting position or climbing up stairs. Weakness continues to progress and involve upper extremities and, in $50 \%$ of cases, involve facial and bulbar muscles which can lead to respiratory failure [168]. Patients can develop profound dysautonomias that can be a cause of mortality during their hospital course. They include labile blood pressures with or without triggers, cardiac arrhythmias with severe bradycardias, Takotsubo cardiomyopathy, adynamic ileus and bladder dysfunction [169-172]. Symptoms reach their peak in 2-4 weak from the initial presentation [167]. Dysautonomia usually resolves prior to improvement in muscle weakness.

Patients with the Miller Fisher variant present with primary involvement of oculomotor muscles, causing ophthalmoplegia, facial and bulbar weakness. They also have associated ataxia, and as in patients with GBS, these patients have decrease or loss of DTR [173]. Secondary to muscular weakness, patients can develop acute respiratory failure.
Approximately $20-30 \%$ of GBS patients end up requiring mechanical ventilation [167].

\subsubsection{Diagnosis}

There are only few other diseases that can be considered in differential diagnosis of GBS with its hallmark ascending paralysis. Transvers myelitis, botulism, MG, severe hypokalemia, and heavy metal intoxication should be considered when patients present with acute muscle weakness. If during physical exam, findings not typically associated with GBS are present (i.e. hyperreflexia or pyramidal signs), a spinal cord MRI should be considered to rule out an alternative cause of symptoms.

CSF analysis often shows an elevated protein with a normal white blood cell count, a finding known as albuminocytologic dissociation. It's important to remember that patients may have a normal protein level and white count if the study is done when muscle weakness is mild. If CSF profile shows elevated levels of white blood cell, infectious or inflammatory diseases like HIV, Lyme, and sarcoidosis should be considered [168].

\subsubsection{NCS/EMG}

NCS in GBS can support the diagnosis and help differentiate between axonal and demyelinating variants of GBS. Early in the course, NCS can be normal. The most prominent abnormalities are seen typically about 2 weeks into the course of the disease [160]. In early stages of AIDP, the most common findings are $\mathrm{F}$ wave abnormalities, decrease in CMAP, and conduction block, which is the most sensitive parameter [174]. In demyelinating form of GBS, findings are increased $F$ wave latency, prolonged distal motor latency, conduction block, and temporal dispersion, while sensory nerve potentials are normal [175]. In axonal forms, NCS shows decreased motor or sensory amplitudes based on the nerves involved. If it is a mixed motor and sensory form, a decrease in both amplitudes will be seen. At times, there may be a transient conduction block, secondary to involvement of nodes of Ranvier in these neurons [176]. This transient conduction block can cause confusion in differentiating between demyelinating form and 
axonal form; however since it is reversible in the axonal variant, repeating NCS later in the course of the disease can help differentiate the two [177].

EMG shows decreased recruitment, presence of fibrillation, positive sharp waves, and polyphasic motor unit action potentials (MUAPs) at different times during the course of the disease. Fibrillations are more prominent between weeks 6 and 10 and MUAPs between week 9 and 15 after the onset of symptoms [178].

\subsubsection{Treatment}

All patients who are diagnosed with GBS should be closely monitored as deterioration from muscle weakness can occur rapidly. If signs of respiratory failure, rapidly progressive weakness, or severe autonomic dysfunction are present, an admission to ICU is warranted for cardiopulmonary and hemodynamic monitoring and support. Immunotherapy with IVIG or PLEX should be initiated as soon as the diagnosis is suspected, and the performance of diagnostic tests should not delay treatment [179].

Two effective disease-modifying treatments that exist for GBS are PLEX and IVIG, and treatment should be initiated with one of these modalities as soon as possible as they hasten recovery. PLEX works by removing antibodies from systemic circulation. It is performed every other day for five courses, and as discussed in the MG section, plasma is substituted with albumin 5\% after each treatment [180]. It has been shown to increase muscle strength and reduce the need for mechanical ventilation [181, 182]. IVIG is pooled $\mathrm{IgG}$ that is administered at $0.4 \mathrm{~g} / \mathrm{kg}$ per day infusion for 5 days. It is as effective as PLEX in treatment of GBS [179], and there is some evidence that it may have fewer complications compared to PLEX [183, 184].

IVIG and PLEX have been shown to be equally effective in treatment of GBS, and the decision on using one or the other is dependent on patient's comorbidities, contraindications, and availability of the treatment [185]. Administration of IVIG and PLEX together has not been shown to be beneficial in treatment of GBS [184]. Corticosteroids have limited to no effect on GBS patients in both acute phase of disease and for long-term outcomes [186].

\subsubsection{Preventative Measures}

Patients with GBS have limited mobility secondary to neuromuscular weakness and spend most of their time immobilized in their bed or chair, which increases their risk of deep venous thromboses (DVT). Therefore, it is crucial for these patients to be placed on intermittent pneumatic compression devices and thromboprophylaxis such as subcutaneous heparin if they do not have contraindications. Pulmonary embolism should be ruled out if patient develops an acute decline in their respiratory status [185]. In patients with corticobulbar weakness, swallowing evaluation should be performed prior to initiation a diet to avoid aspiration.

\subsubsection{Prognosis and Outcome}

The outcome and prognosis of patients with GBS depends on their clinical phenotype. In the purely demyelinating type, remyelination is possible. However, in patients with the purely axonal type, axonal regeneration may not be possible if there is extensive damage to the axon, and even when there is any possibility of regeneration, it is very slow. For this reason, patients with AMAN have longer recovery periods.

Older age, rapid onset, severe muscle weakness on presentation, preceding diarrheal illness, and need for ventilatory support are predictive of poorer recovery from GBS [154, 187, 188]. A patient recovery scoring system, Erasmus GBS Outcome Scale (EGOS), is used to predict patient's ability to ambulate 6 months after GBS onset. The score is performed 2 weeks after admission [189]. EGOS looks at three patient characteristics: age $>40$ years old, history of diarrhea or $C$. jejuni infection in the past 4 weeks, and severe disability at the nadir of the disease.

Approximately $20 \%$ of patients with GBS will not be able to walk without assistance at 6 months, and most patients continue to experience fatigue and pain [190, 191]. Mortality rate has been estimated at 3-7\% [165, 192] in North America and Europe. As deterioration can happen 
quickly in both the early and later courses of disease, prolonged monitoring of patients until they have demonstrated stability is recommended [192, 193].

\subsubsection{Respiratory Failure in Neuromuscular Disorders}

Various neuromuscular diseases can produce severe weakness of respiratory muscles and result in ventilatory failure. This can be acute or be a sudden exacerbation of chronic progressive neuromuscular disease [194]. Patients with neuromuscular respiratory failure without a known diagnosis prior to admission have poorer outcomes, and those whose diagnosis remains unknown at discharge have the highest disability [195]. Therefore, even though these cases often represent a diagnostic challenge in the ICU, evaluation and timely diagnosis of their neuromuscular weakness is essential.

There are four groups of muscles that are primarily involved in ventilation and respiratory efforts:

1. Bulbar muscles: Responsible for maintaining the airway open and assisting with clearing secretions

2. Diaphragm: The main respiratory muscle responsible for $70 \%$ of respiratory function at rest; innervated by phrenic nerve from $\mathrm{C} 3$ to $\mathrm{C} 5$

3. Accessory inspiratory muscles: These consist of external intercostal, scalene, and sternocleidomastoid muscles, which take over more responsibility when there is increased work of breathing

4. Expiratory muscles: These consist of internal intercostal and abdominal wall muscles which help with forced expiration.

\subsubsection{History and Physical Exam}

When a patient presents with acute neuromuscular respiratory failure, a detailed history and a careful neurologic exam are warranted to help establish the underlying cause of respiratory failure. Patients and their families should be asked about any recent infections, surgeries, changes in medications, new or developing symptoms (diplopia, difficulty swallowing, or changes in their voice), and a prior history of similar symptoms or prior hospital administration for respiratory failure requiring mechanical ventilations. The onset of symptoms, the pattern of muscular weakness, and the speed at which they progress are crucial in predicting the need for mechanical ventilation.

On physical exam, dyspnea, tachypnea, tachycardia, staccato speech (inability to finish a sentence in one breath), diaphoresis, use of accessory muscles, weak cough, and, most important of all, paradoxical breathing are signs of impending respiratory failure and should be closely monitored [196]. Patients with neck flexion weakness usually have associated diaphragmatic weakness. Vital capacity can be evaluated by asking the patient to count from 1 to 20 in one single breath. Facial muscles, gag and cough reflex, and tongue strength should be checked which helps evaluate patient's ability to maintain airway and manage secretions. In all patients with neuromuscular weakness, a general exam including vital signs and a heart, lung, and abdominal exam is necessary to evaluate for signs of dysautonomia, cardiopulmonary instability, abdominal distension and/or paradoxical breathing.

\subsubsection{Workup of Respiratory Failure}

After a detailed history and physical exam, patients should be worked up for reversible underlying causes of respiratory failure. All patients with signs of impending respiratory failure should be admitted to an ICU for close monitoring with continuous pulse oximetry. Chest $\mathrm{X}$-ray (CXR) is necessary to identify underlying pulmonary diseases like pneumonia or pulmonary edema which can trigger respiratory failure or worsen respiratory function. Arterial blood gas $(\mathrm{ABG})$ can provide information regarding oxygenation and ventilation by measuring $\mathrm{PaO}_{2}$ and $\mathrm{PaCO}_{2}$, respectively. Worsening of respiratory failure and need for invasive ventilation should be monitored by bedside assessment of forced vital capacity (FVC), maximum inspiratory 
pressure (MIP)/negative inspiratory force (NIF), and maximum expiratory pressure (MEP). FVC of less than $20 \mathrm{ml} / \mathrm{kg}$ or a $30 \%$ decline or more over $24 \mathrm{~h}$ and MIP of less than $-30 \mathrm{~cm} \mathrm{H}_{2} \mathrm{O}$ or MEP of less than $40 \mathrm{~cm} \mathrm{H}_{2} \mathrm{O}$ are indicators of impending respiratory failure requiring intubation [197]. However, one must remember that these tests are effort dependent, can be falsely low if patient is fatigued, and requires that patient receive proper instructions prior to their performance. Moreover, in patients with facial muscle weakness, providing a good seal around the spirometer can be difficult and result in erroneous measurements. It is preferred that these measurements are taken in the same body position every time since FVC and MIP are higher when the patient is in upright position compared to supine position. A decrease of more than $20 \%$ in FVC from upright to supine position is indicative of diaphragm weakness.

\subsubsection{Respiratory Support and Ventilation}

The pattern of respiratory failure seen in NMDs is restrictive. Primarily patients develop microatelectasis at the base of the lungs due to muscular weakness resulting in tachypnea and subsequently respiratory alkalosis. As patients become more fatigued, they develop alveolar hypoventilation, which is evident by continued and worsening tachypnea, normal $\mathrm{PCO}_{2}$, and slightly low levels of $\mathrm{PaO}_{2}$ on $\mathrm{ABG}$. In later stage of respiratory failure with worsening muscular weakness and increasing fatigue, alveoli start to collapse resulting in severe hypoxemia and hypercapnia [194]. These signs and symptoms should alert the physicians to an impending respiratory failure and consideration of mechanical ventilatory support.

Modes of ventilation support can be divided into noninvasive ventilation (NIV) such as CPAP and BiPAP or invasive ventilation, i.e., intubation. Noninvasive modes of ventilation can be beneficial in acute setting in ICU or ED or in late stages of progressive NMDs as seen in ALS. It is of utmost importance that patient's cardiopulmonary comorbidities, mental status, and secretion are evaluated prior to administration of NIV.
Noninvasive ventilation includes continuous positive airway pressure (CPAP) and bilevel positive airway pressure (BiPAP). Both can be used in patients with NMDs. CPAP provides a continuous positive pressure, which helps maintaining the airway and alveoli open; however, CPAP is not as helpful in patients with respiratory muscle fatigue. BiPAP is a great option for patients with respiratory failure secondary to neuromuscular weakness, since it provides positive airway pressure during inspiration and expiration with oxygen flow that helps improve oxygenation and ventilation [198]. It is important to keep in mind that patients with neuromuscular weakness mostly breathe through their mouth so the mask used in noninvasive ventilation should cover both the nose and mouth [194]. One caveat to use of noninvasive mode of ventilation is in patients with bulbar weakness that may be having difficulty in managing their secretions-using positive airway pressure can increase their chances of aspiration.

In patients who present with a MG crisis, NIV can be used as the primary attempt to stabilize patients and has been shown to decrease the length of stay in ICU and rate of intubation in this group. NIV should be initiated as soon as there are signs of respiratory failure and before patient becomes hypercapnic which increases the chances of NIV failure [199, 200]. NIV can also be considered as a rescue therapy in postextubation patients who are showing increased work of breathing and possible need for reintubation [201].

In MG patients who present with impending respiratory failure or require intubation, acetylcholinesterase inhibitors should be discontinued to rule out cholinergic crisis as a possible cause of weakness and respiratory failure. This also helps to decrease salivation in patients who have difficulty managing their secretions. These medications can be restarted after patients' symptoms improve or after extubation [185].

In patient who require intubation, as their symptoms improve, spontaneous breathing trials are recommended, and it is preferred to monitor them for $24 \mathrm{~h}$ on PSV prior to extubation to ensure they do not fatigue. Their cough strength 
should be considered as it is indicative of their ability to maintain their airway open and manage their secretions. Age of over 50 years old, low peak FVC values in the first week, and elevated bicarbonate level at baseline are predictive factors of longer time on mechanical ventilation [86].

Patients with Guillain-Barré syndrome (GBS) who develop respiratory failure as a result of muscular weakness usually continue to worsen as the disease reaches its nadir followed by a prolonged recovery period. Therefore NIV is usually not helpful, and they will require emergent intubation [202]. It is important to remember that these patients develop dysautonomia as part of the disease pathology that can cause labile blood pressure, cardiac arrhythmias, bradycardia, and conduction blocks in response to medication administered during an emergent intubation. It is crucial to closely monitor signs and symptoms of respiratory failure, severe bulbar weakness or rapidly progressive muscular weakness, and consider elective intubation [202]. NIV in patients with GBS can be used in post-extubation period when patients have recovered from their acute phase and are showing enough improvement in muscle strength to be considered appropriate for extubation [201].

Other diseases that can benefit from NIV are progressive NMDs like ALS and muscular dystrophies, when an acute illness causes a sudden decompensation in their respiratory function. If it is determined that underlying disease progression is unlikely and, despite their neuromuscular weakness, they did not require interventions prior to their acute illness, they could benefit from NIV [203, 204].

\subsection{Anesthetic Considerations in Patients with Neuromuscular Disorders}

Anesthetic management of patients with NMDs poses a challenge to the provider given its relatively low incidence, variable pharmacologic response, and presence of multiple coexistent diseases. General anesthesia can exacerbate respiratory and cardiovascular symptoms due to a marked sensitivity to anesthetic drugs. Furthermore, succinylcholine and halogenated volatile agents can trigger life-threatening reactions, such as severe hyperkalemia, rhabdomyolysis, and malignant hyperthermia. In addition, during acute illness, surgery, or ICU admission, patients with neuromuscular diseases are exposed to high levels of physiologic stress response which can exacerbate symptomatology. Cardiac and pulmonary complications represent the major cause of perioperative complications, being respiratory insufficiency the leading cause of death among patients with NMDs [205].

\subsubsection{Preoperative Assessment}

A thorough history and physical examination is a key component of the preoperative evaluation of patients with NMDs. A detailed neurologic examination is essential to confirm the diagnosis and to determine presence of bulbar dysfunction and peripheral and autonomic neuropathy [206]. Patients and family must be made aware of potential risks and complications associated with anesthetic procedures during the intraoperative and postoperative periods.

\subsubsection{Preoperative Pulmonary Evaluation}

Respiratory involvement varies significantly between patients with NMDs [207]. Inspiratory force reduction results in pulmonary disease with a restrictive pattern, with a progressive reduction of forced vital capacity [208]. Hypoventilation, atelectasis, hypoxia, and inability to clear airway secretions can be seen with more advanced inspiratory muscle disease. Inability to swallow can be seen with bulbar involvement, which is especially high in patients with ALS and MG [209, 210]. Detailed preoperative evaluation is recommended in patients with advanced disease to assess the risk of respiratory complications and the need for perioperative optimization [211-213]. In addition to medical history and physical examination, preoperative workup should include CXR, ABG, and pulmonary function tests (PFTs) when 
feasible [211-213]. When PFTs are abnormal, NIV and manual or mechanically assisted cough techniques may be indicated. NIV is recommended in patients with Duchenne muscular dystrophy with FVC $<50 \%$ [214].

In addition to the regular preoperative airway evaluation, careful assessment for macroglossia, mandible ankylosis, atrophy of masseter muscles, and limited cervical spine range of motion must be performed [215, 216]. Providers should follow the current guidelines for management of difficult airway when encountered with these findings.

\subsubsection{Cardiac Preoperative Evaluation}

Cardiac evaluation is another key component of patients with NMDs, as they can manifest with myocardial failure, arrhythmias, and autonomic dysfunction leading to inability to modify cardiac output in response to surgical stress and currently used anesthetics. Patients with evidence of nocturnal hypoxia can present with right ventricular dysfunction resultant from pulmonary hypertension [215]. Moreover, relative immobility of patients with NMDs may predispose development of deep venous thrombosis and pulmonary embolism perioperatively. Workup should include electrocardiogram (ECG) and serial echocardiograms in all patients when possible to assess fractional shortening and left ventricular ejection fraction [217-219]. Characteristic ECG findings related to fibrous replacement of the myocardium include tall right precordial $\mathrm{R}$ and $\mathrm{Q}$ waves in leads I, aVL, $\mathrm{V}_{5}$, and $\mathrm{V}_{6}$ [220]. $24 \mathrm{~h}$ Holter testing should be considered when there is suspicion for arrhythmias [218, 219].

\subsubsection{Miscellaneous Preoperative Anesthetic Considerations}

Patients with NMDs are at increased risk of intraoperative positioning injuries related to chronic contractures and skeletal deformities. These can be prevented with careful padding and positioning of extremities, head, and torso. When possible, nutritional status should be optimized before surgery to favor adequate wound healing [211, 212]. Premedication should be given carefully as there is an increased sensitivity to narcotics and benzodiazepines, leading to sleep apnea and hypoventilation [216]. Perioperative stress dose steroid administration should be considered in NMD patients that take steroids chronically.

In addition to the standard American Society of Anesthesiologists (ASA) monitors, an arterial line should be placed when there is suspicion or evidence of cardiac compromise. Careful temperature monitoring is crucial as NMDs patients are predispose to hypothermia [206] and/or hyperthermia which can trigger disease flares. Intravenous access can be challenging, and ultrasound-guided techniques should be used when possible to limit unnecessary discomfort [221].

\subsubsection{Intraoperative Management}

The anesthetic concerns for specific NMDs are summarized in Table 5.3. In general, the severity of the disease tends to correlate with the incidence of intraoperative complications. In patients with compromised respiratory function, regional anesthesia should be favored over general anesthesia (GA). If regional anesthesia is not an option, GA must be administered avoiding depolarizing neuromuscular blockers and halogenated agents. The cytoskeleton of the muscle membrane is abnormal in patients with NMDs, and life-threatening amounts of intracellular potassium can be released after exposure to succinylcholine and volatile agents. Total intravenous anesthesia based on short-acting opioids, propofol, and dexmedetomidine should be a firstline option, considering their cardiac depressant effects.

In most patients with NMDs, nondepolarizing neuromuscular blockers may show prolonged duration of blockade. Therefore, there is a strong recommendation to avoid its use intraoperatively whenever possible [222, 223]. If the use of neuromuscular blockers is needed, smaller doses should be titrated to effect using train-of-four monitoring [216, 224]. Rapid sequence induction can be performed safely with high-dose 


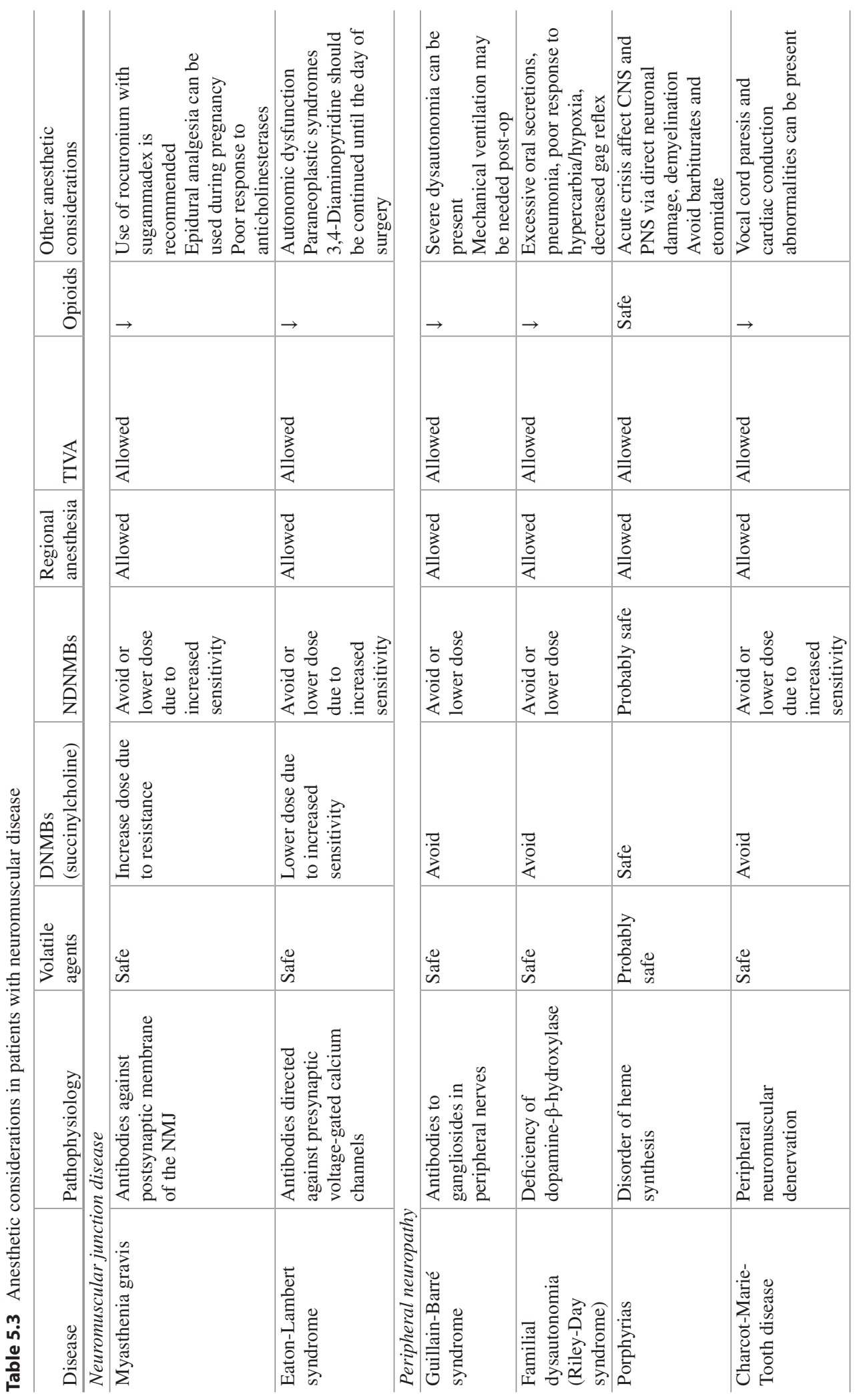




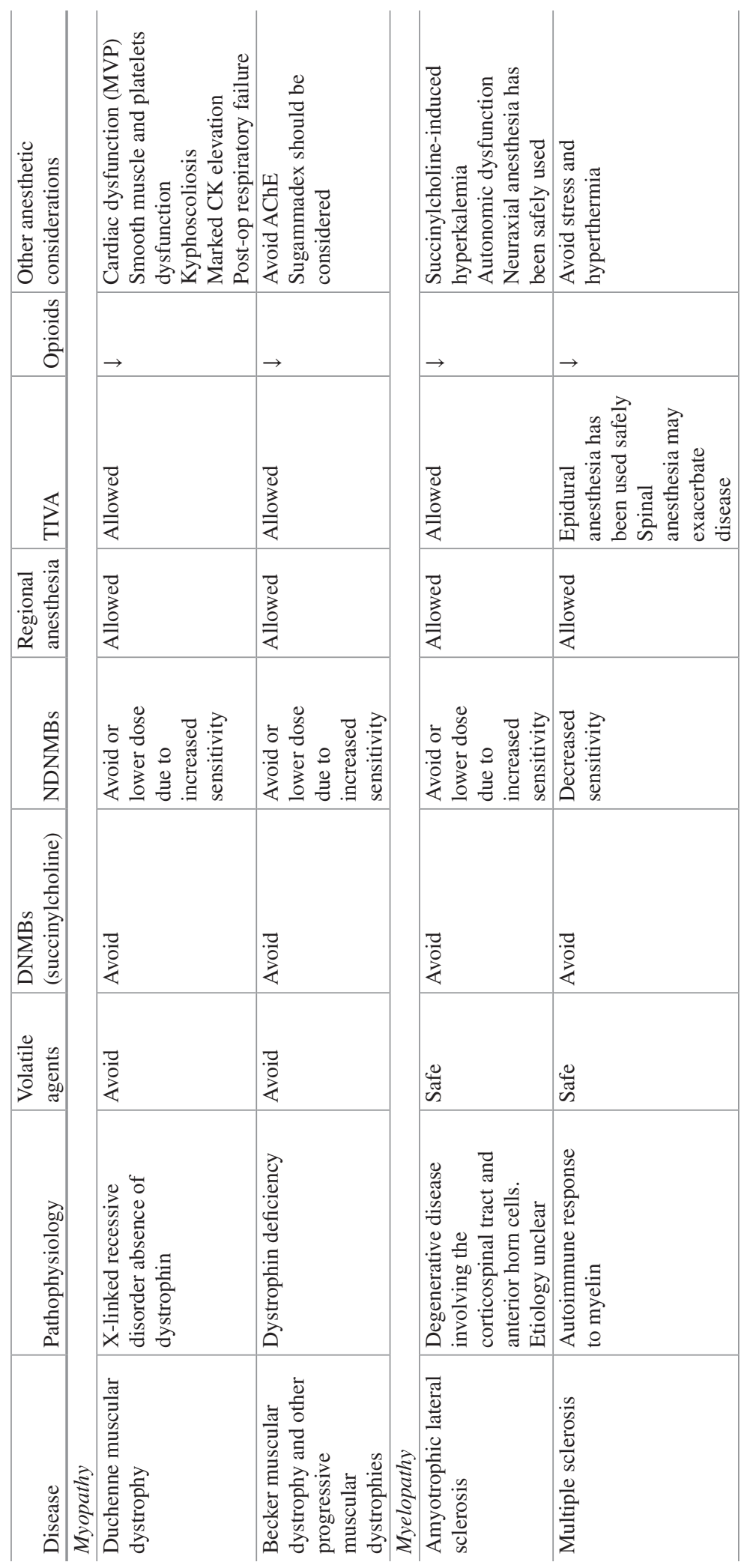




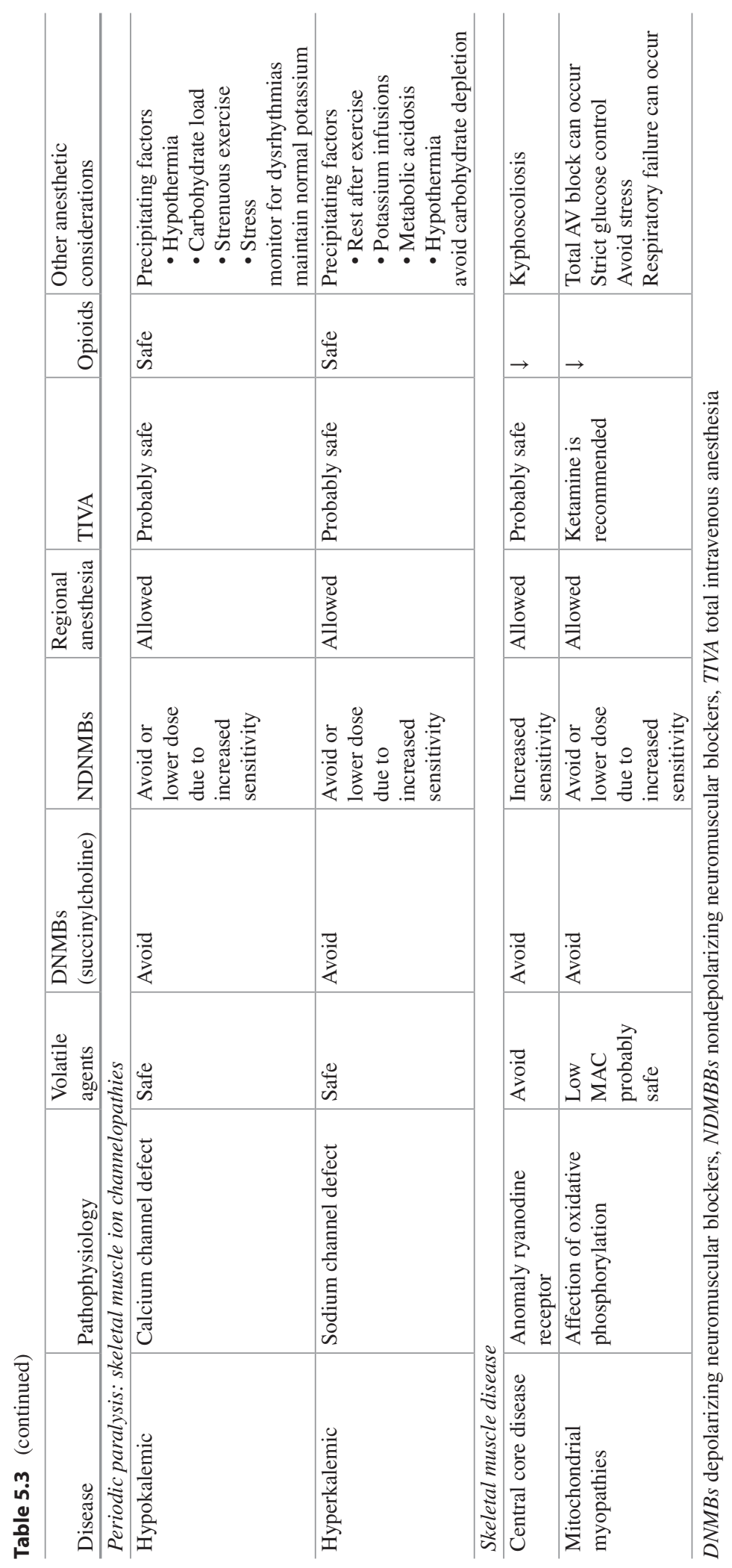


rocuronium, which can be quickly reversed with the now FDA-approved sugammadex [225, 226].

The use of regional anesthesia offers a significant advantage in patients at risk for postoperative respiratory complications $[214,217]$. On the other hand, it has some potential risks especially in patients with pre-existing peripheral neuropathy who are at increased risk of suffering from permanent neurologic damage from inadvertent nerve puncture, local anesthetic, and vasopressors use. However, evidence suggests that the use of ultrasound-guided nerve blocks significantly reduced amount of local anesthetic and major vascular complications [227].

\subsubsection{Postoperative ICU Management}

Hypoventilation is a major postoperative risk in patients with NMDs. Therefore, careful titration of analgesia is crucial in preventing depressed respiratory function [213, 228]. Narcotics should be administered in conjunction with preemptive analgesia (acetaminophen, NSAIDs, gabapentin, clonidine, etc.) to reduce opioid requirements [229, 230]. Also, peripheral nerve blocks and wound infiltration with local anesthetics should be used when possible to achieve adequate analgesia without the need of excessive opioid administration.

Postoperative extubation success should be individualized based on preoperative pulmonary function [213]. Extubation to NIV should be considered for patients with a baseline FVC less than $50 \%$ of predicted. Postoperative use of assisted cough techniques such as mechanical insufflators/exsufflator must be considered for any patient with preoperative maximum expiratory force (MEP) $<60 \mathrm{~cm} \mathrm{H}_{2} \mathrm{O}$ [228]. If excessive oral secretions are a concern, extubation should be delayed in order to decrease the risk of aspiration and reintubation [231].

In conclusion, NMDs represent a heterogeneous spectrum of challenges for the anesthesiologist and intensivist. A thorough preoperative evaluation and a detailed knowledge of individual diseases are vital for safe anesthetic management.

\subsection{Conclusion}

Most patients with neuromuscular disorders are diagnosed and treated in the outpatient clinic; however a small but significant subset of these patients can present with a rapidly advancing muscle weakness which can be a life-threatening emergency. The most significant compromise occurs in respiratory function needing mechanical ventilation although monitoring of cardiac function, hemodynamics, and bulbar function is also sometimes warranted. A thorough history and physical examination along with prudent intensive care management has the potential to mitigate morbidity and mortality. With the increased availability of therapies such as immunoglobulins, plasma exchange, steroid-sparing agents, immunomodulatory therapy, as well as specialized care in neurointensive care units, physicians today have a range of therapies to offer.

\section{Key Points}

- Neuromuscular conditions are diseases of the nervous system that affect the motor neuron unit between the anterior horn cells and the muscle.

- Anesthetic management of patients with NMDs poses a challenge to the provider given its relatively low incidence, variable pharmacologic response, and presence of multiple coexistent diseases.

- General anesthesia can exacerbate respiratory and cardiovascular symptoms due to a marked sensitivity to anesthetic drugs.

- Hypoventilation is a major postoperative risk in patients with NMDs.

- With the increased availability of therapies such as immunoglobulins, plasma exchange, steroid-sparing agents, immunomodulatory therapy, as well as specialized care in neurocritical care units, physicians today have a range of therapies to offer. 


\section{References}

1. Walton J, Thomas PK. Classification of neuromuscular diseases. J Neurol Sci. 1988;86(2):333-60.

2. Fardeau M, Desguerre I. Diagnostic workup for neuromuscular diseases. Handb Clin Neurol. 2013;113:1291-7.

3. Targoff IN. Laboratory testing in the diagnosis and management of idiopathic inflammatory myopathies. Rheum Dis Clin N Am. 2002;28(4):859-90.

4. Targoff IN. Laboratory manifestations of polymyositis/dermatomyositis. Clin Dermatol. 1988;6(2):76-92.

5. Ropper AH, Samuels MAS. Chapter 45. Electrophysiologic and laboratory aids in the diagnosis of neuromuscular disease. In: Adams and Victor's principles of neurology. 9th ed. New York: McGraw-Hill; 2009.

6. Selvan VA, Single-fiber EMG. A review. Ann Indian Acad Neurol. 2011;14(1):64-7.

7. Barboi AC, Barkhaus PE. Electrodiagnostic testing in neuromuscular disorders. Neurol Clin. 2004;22(3):619-41. vi.

8. Lipa BM, Han JJ. Electrodiagnosis in neuromuscular disease. Phys Med Rehabil Clin N Am. 2012;23(3):565-87.

9. Fournier E, et al. Electromyography guides toward subgroups of mutations in muscle channelopathies. Ann Neurol. 2004;56(5):650-61.

10. Preston DC, Shapiro BE. Needle electromyography. Fundamentals, normal and abnormal patterns. Neurol Clin. 2002;20(2):361-96.. vi

11. Paganoni S, Amato A. Electrodiagnostic evaluation of myopathies. Phys Med Rehabil Clin N Am. 2013;24(1):193-207.

12. May DA, et al. Abnormal signal intensity in skeletal muscle at MR imaging: patterns, pearls, and pitfalls. Radiographics. 2000;20:S295-315. Spec No.

13. Tasca G, et al. Magnetic resonance imaging pattern recognition in sporadic inclusion-body myositis. Muscle Nerve. 2015;52(6):956-62.

14. Fleckenstein JL. MRI of neuromuscular disease: the basics. Semin Musculoskelet Radiol. 2000;4(04):393-420.

15. Shaibani A, et al. Diagnostic outcome of muscle biopsy. Muscle Nerve. 2015;51(5):662-8.

16. Mikell CB, et al. Muscle and nerve biopsies: techniques for the neurologist and neurosurgeon. Clin Neurol Neurosurg. 2013;115(8):1206-14.

17. DiMauro SS. Metabolic myopathies. Curr Rheumatol Rep. 2010;12(5):386-93.

18. Bolton CF, et al. Polyneuropathy in critically ill patients. J Neurol Neurosurg Psychiatry. 1984; 47(11):1223-31.

19. Segredo V, et al. Persistent paralysis in critically ill patients after long-term administration of vecuronium. N Engl J Med. 1992;327(8):524-8.

20. Hirano M, et al. Acute quadriplegic myopathy: a complication of treatment with steroids, nondepolarizing blocking agents, or both. Neurology. 1992;42(11):2082-7.
21. Latronico N, et al. Critical illness myopathy and neuropathy. Lancet. 1996;347(9015):1579-82.

22. Latronico N, Bolton CF. Critical illness polyneuropathy and myopathy: a major cause of muscle weakness and paralysis. Lancet Neurol. 2011;10(10):931-41.

23. Stevens RD, et al. A framework for diagnosing and classifying intensive care unit-acquired weakness. Crit Care Med. 2009;37(10 Suppl):S299-308.

24. Schweickert WD, Hall J. ICU-acquired weakness. Chest. 2007;131(5):1541-9.

25. Latronico N, Peli E, Botteri M. Critical illness myopathy and neuropathy. Curr Opin Crit Care. 2005;11(2):126-32.

26. De Jonghe $\mathrm{B}$, et al. Paresis acquired in the intensive care unit: a prospective multicenter study. JAMA. 2002;288(22):2859-67.

27. Bercker S, et al. Critical illness polyneuropathy and myopathy in patients with acute respiratory distress syndrome. Crit Care Med. 2005;33(4):711-5.

28. Hermans G, et al. Impact of intensive insulin therapy on neuromuscular complications and ventilator dependency in the medical intensive care unit. Am J Respir Crit Care Med. 2007;175(5):480-9.

29. Coakley JH, et al. Patterns of neurophysiological abnormality in prolonged critical illness. Intensive Care Med. 1998;24(8):801-7.

30. Tennila A, et al. Early signs of critical illness polyneuropathy in ICU patients with systemic inflammatory response syndrome or sepsis. Intensive Care Med. 2000;26(9):1360-3.

31. Zochodne DW, et al. Critical illness polyneuropathy. A complication of sepsis and multiple organ failure. Brain. 1987;110(Pt 4):819-41.

32. Bednarik J, et al. Risk factors for critical illness polyneuromyopathy. J Neurol. 2005;252(3):343-51.

33. de Letter MA, et al. Risk factors for the development of polyneuropathy and myopathy in critically ill patients. Crit Care Med. 2001;29(12):2281-6.

34. Jaber S, et al. Rapidly progressive diaphragmatic weakness and injury during mechanical ventilation in humans. Am J Respir Crit Care Med. 2011;183(3):364-71.

35. Piper RD, et al. Microcirculatory changes in rat skeletal muscle in sepsis. Am J Respir Crit Care Med. 1996;154(4 Pt 1):931-7.

36. Fink MP, Evans TW. Mechanisms of organ dysfunction in critical illness: report from a round table conference held in Brussels. Intensive Care Med. 2002;28(3):369-75.

37. Brealey D, et al. Association between mitochondrial dysfunction and severity and outcome of septic shock. Lancet. 2002;360(9328):219-23.

38. Bolton CF. Neuromuscular manifestations of critical illness. Muscle Nerve. 2005;32(2):140-63.

39. Fenzi F, et al. Enhanced expression of E-selectin on the vascular endothelium of peripheral nerve in critically ill patients with neuromuscular disorders. Acta Neuropathol. 2003;106(1):75-82.

40. Witt NJ, et al. Peripheral nerve function in sepsis and multiple organ failure. Chest. 1991;99(1):176-84. 
41. Van den Berghe $\mathrm{G}$, et al. Insulin therapy protects the central and peripheral nervous system of intensive care patients. Neurology. 2005;64(8):1348-53.

42. Garnacho-Montero J, et al. Critical illness polyneuropathy: risk factors and clinical consequences. A cohort study in septic patients. Intensive Care Med. 2001;27(8):1288-96.

43. Minetti $\mathrm{C}$, et al. Ubiquitin expression in acute steroid myopathy with loss of myosin thick filaments. Muscle Nerve. 1996;19(1):94-6.

44. Papazian L, et al. Neuromuscular blockers in early acute respiratory distress syndrome. N Engl J Med. 2010;363(12):1107-16.

45. Murray MJ, Brull SJ, Bolton CF. Brief review: nondepolarizing neuromuscular blocking drugs and critical illness myopathy. Can J Anaesth. 2006;53(11):1148-56.

46. Kress JP, Hall JB. ICU-acquired weakness and recovery from critical illness. N Engl J Med. 2014; 371(3):287-8.

47. Danon MJ, Carpenter S. Myopathy with thick filament (myosin) loss following prolonged paralysis with vecuronium during steroid treatment. Muscle Nerve. 1991;14(11):1131-9.

48. Zifko UA, Zipko HT, Bolton CF. Clinical and electrophysiological findings in critical illness polyneuropathy. J Neurol Sci. 1998;159(2):186-93.

49. Lacomis D. Electrophysiology of neuromuscular disorders in critical illness. Muscle Nerve. 2013; 47(3):452-63.

50. Park EJ, et al. Prolonged compound muscle action potential duration in critical illness myopathy: report of nine cases. J Clin Neuromuscul Dis. 2004;5(4):176-83.

51. Rabinstein AA. Acute neuromuscular respiratory failure. Continuum Lifelong Learn Neurol. 2015; 21(5, Neurocritical Care):1324-45.

52. Bolton CF. AAEM minimonograph \#40: clinical neurophysiology of the respiratory system. Muscle Nerve. 1993;16(8):809-18.

53. Hermans $\mathrm{G}$, et al. Interventions for preventing critical illness polyneuropathy and critical illness myopathy. Cochrane Database Syst Rev. 2009; 1:Cd006832.

54. Schweickert WD, et al. Early physical and occupational therapy in mechanically ventilated, critically ill patients: a randomised controlled trial. Lancet. 2009;373(9678):1874-82.

55. Finfer $\mathrm{S}$, et al. Intensive versus conventional glucose control in critically ill patients. N Engl J Med. 2009;360(13):1283-97.

56. Griffiths RD, et al. Effect of passive stretching on the wasting of muscle in the critically ill. Nutrition. 1995;11(5):428-32.

57. Guarneri B, Bertolini G, Latronico N. Long-term outcome in patients with critical illness myopathy or neuropathy: the Italian multicentre CRIMYNE study. J Neurol Neurosurg Psychiatry. 2008;79(7): 838-41.
58. Santos CD, et al. Mechanisms of chronic muscle wasting and dysfunction after an intensive care unit stay. A pilot study. Am J Respir Crit Care Med. 2016;194(7):821-30.

59. Rocheteau P, et al. Sepsis induces long-term metabolic and mitochondrial muscle stem cell dysfunction amenable by mesenchymal stem cell therapy. Nat Commun. 2015;6:10145.

60. Ochala J, et al. Factors underlying the early limb muscle weakness in acute quadriplegic myopathy using an experimental ICU porcine model. PLoS One. 2011;6(6):e20876.

61. Corpeno Kalamgi R, et al. Mechano-signalling pathways in an experimental intensive critical illness myopathy model. J Physiol. 2016;594(15):4371-88.

62. Statland JM, Ciafaloni E. Myasthenia gravis: five new things. Neurol Clin Pract. 2013;3(2):126-33.

63. Marsteller HB. The first American case of myasthenia gravis. Arch Neurol. 1988;45(2):185-7.

64. Pascuzzi RM. The history of myasthenia gravis. Neurol Clin. 1994;12(2):231-42.

65. Patrick J, Lindstrom J. Autoimmune response to acetylcholine receptor. Science. 1973;180(4088): 871-2.

66. Carr AS, et al. A systematic review of population based epidemiological studies in myasthenia gravis. BMC Neurol. 2010;10:46.

67. Hemminki K, Li X, Sundquist K. Familial risks for diseases of myoneural junction and muscle in siblings based on hospitalizations and deaths in Sweden. Twin Res Hum Genet. 2006;9(4):573-9.

68. Boldingh MI, et al. Increased risk for clinical onset of myasthenia gravis during the postpartum period. Neurology. 2016;87(20):2139-45.

69. Tzartos SJ, Seybold ME, Lindstrom JM. Specificities of antibodies to acetylcholine receptors in sera from myasthenia gravis patients measured by monoclonal antibodies. Proc Natl Acad Sci U S A. 1982;79(1):188-92.

70. Tzartos SJ, et al. Anatomy of the antigenic structure of a large membrane autoantigen, the muscletype nicotinic acetylcholine receptor. Immunol Rev. 1998;163:89-120.

71. Benatar M. A systematic review of diagnostic studies in myasthenia gravis. Neuromuscul Disord. 2006;16(7):459-67.

72. Tsonis AI, et al. MuSK autoantibodies in myasthenia gravis detected by cell based assay--a multinational study. J Neuroimmunol. 2015;284:10-7.

73. Engel AG. Myasthenia gravis and myasthenic syndromes. Ann Neurol. 1984;16(5):519-34.

74. Engel AG, Arahata K. The membrane attack complex of complement at the endplate in myasthenia gravis. Ann N Y Acad Sci. 1987;505:326-32.

75. Drachman DB, et al. Myasthenic antibodies crosslink acetylcholine receptors to accelerate degradation. N Engl J Med. 1978;298(20):1116-22.

76. Luo J, Lindstrom J. AChR-specific immunosuppressive therapy of myasthenia gravis. Biochem Pharmacol. 2015;97(4):609-19. 
77. Hoch W, et al. Auto-antibodies to the receptor tyrosine kinase MuSK in patients with myasthenia gravis without acetylcholine receptor antibodies. Nat Med. 2001;7(3):365-8.

78. Hurst RL, Gooch CL. Muscle-specific receptor tyrosine kinase (MuSK) myasthenia gravis. Curr Neurol Neurosci Rep. 2016;16(7):61.

79. Koneczny I, Cossins J, Vincent A. The role of muscle-specific tyrosine kinase (MuSK) and mystery of MuSK myasthenia gravis. J Anat. 2014;224(1): 29-35.

80. Zhang B, et al. Autoantibodies to lipoprotein-related protein 4 in patients with double-seronegative myasthenia gravis. Arch Neurol. 2012;69(4):445-51.

81. Wong SH, et al. Ocular myasthenia gravis: controversies and updates. Curr Neurol Neurosci Rep. 2014;14(1):421.

82. Bever CT Jr, et al. Prognosis of ocular myasthenia. Ann Neurol. 1983;14(5):516-9.

83. Grob D, et al. Lifetime course of myasthenia gravis. Muscle Nerve. 2008;37(2):141-9.

84. Oosterhuis HJ. The natural course of myasthenia gravis: a long term follow up study. J Neurol Neurosurg Psychiatry. 1989;52(10):1121-7.

85. Sanders DB, et al. Clinical aspects of MuSK antibody positive seronegative MG. Neurology. 2003;60(12):1978-80.

86. Thomas CE, et al. Myasthenic crisis: clinical features, mortality, complications, and risk factors for prolonged intubation. Neurology. 1997;48(5):1253-60.

87. Kalita J, Kohat AK, Misra UK. Predictors of outcome of myasthenic crisis. Neurol Sci. 2014;35(7): 1109-14.

88. Witoonpanich R, et al. Electrophysiological and immunological study in myasthenia gravis: diagnostic sensitivity and correlation. Clin Neurophysiol. 2011;122(9):1873-7.

89. Pascuzzi RM. The edrophonium test. Semin Neurol. 2003;23(1):83-8.

90. Sethi KD, Rivner MH, Swift TR. Ice pack test for myasthenia gravis. Neurology. 1987;37(8):1383-5.

91. Golnik KC, et al. An ice test for the diagnosis of myasthenia gravis. Ophthalmology. 1999;106(7): 1282-6.

92. Alkhawajah NM, Oger J. Treatment of myasthenia gravis in the aged. Drugs Aging. 2015;32(9):689-97.

93. Evoli A, et al. Response to therapy in myasthenia gravis with anti-MuSK antibodies. Ann N Y Acad Sci. 2008;1132:76-83.

94. Melzer N, et al. Clinical features, pathogenesis, and treatment of myasthenia gravis: a supplement to the guidelines of the German neurological society. J Neurol. 2016;263(8):1473-94.

95. Arsura E, et al. High-dose intravenous methylprednisolone in myasthenia gravis. Arch Neurol. 1985;42(12):1149-53.

96. Lindberg C, Andersen O, Lefvert AK. Treatment of myasthenia gravis with methylprednisolone pulse: a double blind study. Acta Neurol Scand. 1998;97(6):370-3.
97. Seybold ME, Drachman DB. Gradually increasing doses of prednisone in myasthenia gravis. Reducing the hazards of treatment. N Engl J Med. 1974;290(2):81-4.

98. Bae JS, Go SM, Kim BJ. Clinical predictors of steroid-induced exacerbation in myasthenia gravis. J Clin Neurosci. 2006;13(10):1006-10.

99. Pascuzzi RM, Coslett HB, Johns TR. Long-term corticosteroid treatment of myasthenia gravis: report of 116 patients. Ann Neurol. 1984;15(3):291-8.

100. Hart IK, Sathasivam S, Sharshar T. Immunosuppressive agents for myasthenia gravis. Cochrane Database Syst Rev. 2007;4:CD005224.

101. Evoli A, et al. Clinical characteristics and prognosis of myasthenia gravis in older people. J Am Geriatr Soc. 2000;48(11):1442-8.

102. O'Donovan P, et al. Azathioprine and UVA light generate mutagenic oxidative DNA damage. Science. 2005;309(5742):1871-4.

103. Michels M, et al. Myasthenia gravis: discontinuation of long-term azathioprine. Ann Neurol. 1988;24(6):798.

104. Hohlfeld R, et al. Myasthenia gravis: reactivation of clinical disease and of autoimmune factors after discontinuation of long-term azathioprine. Ann Neurol. 1985;17(3):238-42.

105. Hohlfeld R, et al. Azathioprine toxicity during longterm immunosuppression of generalized myasthenia gravis. Neurology. 1988;38(2):258-61.

106. Tindall RS, et al. A clinical therapeutic trial of cyclosporine in myasthenia gravis. Ann N Y Acad Sci. 1993;681:539-51.

107. Heckmann JM, et al. A single-blinded trial of methotrexate versus azathioprine as steroid-sparing agents in generalized myasthenia gravis. BMC Neurol. 2011;11:97.

108. Hilton-Jones D. When the patient fails to respond to treatment: myasthenia gravis. Pract Neurol. 2007;7(6):405-11.

109. Ciafaloni E, et al. Mycophenolate mofetil for myasthenia gravis: an open-label pilot study. Neurology. 2001;56(1):97-9.

110. Hanisch F, Wendt M, Zierz S. Mycophenolate mofetil as second line immunosuppressant in myasthenia gravis - a long-term prospective open-label study. Eur J Med Res. 2009;14(8):364.

111. Hehir MK, et al. Mycophenolate mofetil in AChRantibody-positive myasthenia gravis: outcomes in 102 patients. Muscle Nerve. 2010;41(5):593-8.

112. Muscle Study Group. A trial of mycophenolate mofetil with prednisone as initial immunotherapy in myasthenia gravis. Neurology. 2008;71(6):394-9.

113. Sanders DB, et al. An international, phase III, randomized trial of mycophenolate mofetil in myasthenia gravis. Neurology. 2008;71(6):400-6.

114. Evoli A, et al. Successful treatment of myasthenia gravis with tacrolimus. Muscle Nerve. 2002;25(1):111-4.

115. Konishi $\mathrm{T}$, et al. Long-term treatment of generalised myasthenia gravis with FK506 (tacrolimus). J Neurol Neurosurg Psychiatry. 2005;76(3): 448-50. 
116. Minami N, et al. Five-year follow-up with low-dose tacrolimus in patients with myasthenia gravis. J Neurol Sci. 2011;300(1-2):59-62.

117. Nagaishi A, Yukitake M, Kuroda Y. Long-term treatment of steroid-dependent myasthenia gravis patients with low-dose tacrolimus. Intern Med. 2008;47(8):731-6.

118. Ponseti JM, et al. Long-term results of tacrolimus in cyclosporine- and prednisone-dependent myasthenia gravis. Neurology. 2005;64(9):1641-3.

119. Ponseti JM, et al. Tacrolimus for myasthenia gravis: a clinical study of 212 patients. Ann N Y Acad Sci. 2008;1132:254-63.

120. Iorio R, et al. Efficacy and safety of rituximab for myasthenia gravis: a systematic review and metaanalysis. J Neurol. 2015;262(5):1115-9.

121. Diaz-Manera J, et al. Long-lasting treatment effect of rituximab in MuSK myasthenia. Neurology. 2012;78(3):189-93.

122. Huang H, Benoist C, Mathis C. Rituximab specifically depletes short-lived autoreactive plasma cells in a mouse model of inflammatory arthritis. Proc Natl Acad Sci U S A. 2010;107(10):4658-63.

123. Drachman DB, et al. Rebooting the immune system with high-dose cyclophosphamide for treatment of refractory myasthenia gravis. Ann N Y Acad Sci. 2008;1132:305-14.

124. De Feo LG, et al. Use of intravenous pulsed cyclophosphamide in severe, generalized myasthenia gravis. Muscle Nerve. 2002;26(1):31-6.

125. Gladstone DE, et al. High dose cyclophosphamide for severe refractory myasthenia gravis. J Neurol Neurosurg Psychiatry. 2004;75(5):789-91.

126. Strober J, Cowan MJ, Horn BN. Allogeneic hematopoietic cell transplantation for refractory myasthenia gravis. Arch Neurol. 2009;66(5):659-61.

127. Imbach $P$, et al. High-dose intravenous gammaglobulin for idiopathic thrombocytopenic purpura in childhood. Lancet. 1981;1(8232):1228-31.

128. Gajdos P, et al. Treatment of myasthenia gravis exacerbation with intravenous immunoglobulin: a randomized double-blind clinical trial. Arch Neurol. 2005;62(11):1689-93.

129. Gajdos P, Chevret S, Toyka KV. Intravenous immunoglobulin for myasthenia gravis. Cochrane Database Syst Rev. 2012;12:CD002277.

130. Dau PC, et al. Plasmapheresis and immunosuppressive drug therapy in myasthenia gravis. $\mathrm{N}$ Engl $\mathrm{J}$ Med. 1977;297(21):1134-40.

131. Newsom-Davis J, et al. Plasmapheresis for myasthenia gravis. N Engl J Med. 1978;298(8):456-7.

132. Gajdos P, Chevret S, Toyka K. Plasma exchange for myasthenia gravis. Cochrane Database Syst Rev. 2002;4:CD002275.

133. Gajdos P, et al. Clinical trial of plasma exchange and high-dose intravenous immunoglobulin in myasthenia gravis. Myasthenia gravis clinical study group. Ann Neurol. 1997;41(6):789-96.

134. Eienbroker C, et al. Intravenous immunoglobulin maintenance treatment in myasthenia gravis: a ran- domized, controlled trial sample size simulation. Muscle Nerve. 2014;50(6):999-1004.

135. Qureshi AI, et al. Plasma exchange versus intravenous immunoglobulin treatment in myasthenic crisis. Neurology. 1999;52(3):629-32.

136. Stangel M, Gold R. Administration of intravenous immunoglobulins in neurology. An evidence-based consensus: update 2010. Nervenarzt. 2011;82(4): 415-6, 418, 420 passim.

137. Silvestri NJ, Wolfe GI. Myasthenia gravis. Semin Neurol. 2012;32(3):215-26.

138. Kim JY, Park KD, Richman DP. Treatment of myasthenia gravis based on its immunopathogenesis. J Clin Neurol. 2011;7(4):173-83.

139. Wolfe GI, et al. Randomized trial of Thymectomy in myasthenia gravis. N Engl J Med. 2016;375(6): 511-22.

140. Sussman J, et al. Myasthenia gravis: Association of British Neurologists' management guidelines. Pract Neurol. 2015;15(3):199-206.

141. Guptill JT, Sanders DB, Evoli A. Anti-MuSK antibody myasthenia gravis: clinical findings and response to treatment in two large cohorts. Muscle Nerve. 2011;44(1):36-40.

142. Zhou L, et al. Clinical comparison of musclespecific tyrosine kinase (MuSK) antibody-positive and -negative myasthenic patients. Muscle Nerve. 2004;30(1):55-60.

143. Lauriola L, et al. Thymus changes in anti-MuSKpositive and -negative myasthenia gravis. Neurology. 2005;64(3):536-8.

144. Leite MI, et al. Fewer thymic changes in MuSK antibody-positive than in MuSK antibody-negative MG. Ann Neurol. 2005;57(3):444-8.

145. Evoli A, et al. Clinical correlates with anti-MuSK antibodies in generalized seronegative myasthenia gravis. Brain. 2003;126(Pt 10):2304-11.

146. Sejvar JJ, et al. Population incidence of GuillainBarre syndrome: a systematic review and metaanalysis. Neuroepidemiology. 2011;36(2):123-33.

147. Willison HJ, Jacobs BC, van Doorn PA. GuillainBarre syndrome. Lancet. 2016;388(10045):717-27.

148. Frenzen PD. Hospital admissions for GuillainBarre syndrome in the United States, 1993-2004. Neuroepidemiology. 2007;29(1-2):83-8.

149. Huang WC, Lu CL, Chen SC. A 15 -year nationwide epidemiological analysis of Guillain-Barre syndrome in Taiwan. Neuroepidemiology. 2015;44(4):249-54.

150. Lyu RK, et al. Guillain-Barre syndrome in Taiwan: a clinical study of 167 patients. J Neurol Neurosurg Psychiatry. 1997;63(4):494-500.

151. Jacobs BC, et al. The spectrum of antecedent infections in Guillain-Barre syndrome: a case-control study. Neurology. 1998;51(4):1110-5.

152. McCarthy N, et al. The risk of Guillain-Barre syndrome following infection with campylobacter jejuni. Epidemiol Infect. 1999;122(1):15-7.

153. Mori M, et al. Haemophilus influenzae infection and Guillain-Barre syndrome. Brain. 2000;123(Pt 10): 2171-8. 
154. Rees JH, et al. Campylobacter jejuni infection and Guillain-Barre syndrome. $N$ Engl J Med. 1995;333(21):1374-9.

155. Hughes RA, Cornblath DR. Guillain-Barre syndrome. Lancet. 2005;366(9497):1653-66.

156. van den Berg B, et al. Guillain-Barre syndrome: pathogenesis, diagnosis, treatment and prognosis. Nat Rev Neurol. 2014;10(8):469-82.

157. Willison HJ, Goodyear CS. Glycolipid antigens and autoantibodies in autoimmune neuropathies. Trends Immunol. 2013;34(9):453-9.

158. Ho TW, et al. Anti-GDla antibody is associated with axonal but not demyelinating forms of GuillainBarre syndrome. Ann Neurol. 1999;45(2):168-73.

159. Jacobs BC, et al. Campylobacter jejuni infections and anti-GM1 antibodies in Guillain-Barre syndrome. Ann Neurol. 1996;40(2):181-7.

160. Hadden RD, et al. Electrophysiological classification of Guillain-Barre syndrome: clinical associations and outcome. Plasma exchange/Sandoglobulin Guillain-Barre syndrome trial group. Ann Neurol. 1998;44(5):780-8.

161. Jacobs BC, et al. Subclass IgG to motor gangliosides related to infection and clinical course in Guillain-Barre syndrome. J Neuroimmunol. 2008;194(1-2):181-90.

162. McGonigal R, et al. Anti-GD1a antibodies activate complement and calpain to injure distal motor nodes of Ranvier in mice. Brain. 2010;133(Pt 7):1944-60.

163. Plomp JJ, Willison HJ. Pathophysiological actions of neuropathy-related anti-ganglioside antibodies at the neuromuscular junction. J Physiol. 2009;587(Pt 16):3979-99.

164. Liu JX, Willison HJ, Pedrosa-Domellof F. Immunolocalization of GQ1b and related gangliosides in human extraocular neuromuscular junctions and muscle spindles. Invest Ophthalmol Vis Sci. 2009;50(7):3226-32.

165. Islam Z, et al. Axonal variant of Guillain-Barre syndrome associated with campylobacter infection in Bangladesh. Neurology. 2010;74(7):581-7.

166. Makowska A, et al. Immune responses to myelin proteins in Guillain-Barre syndrome. J Neurol Neurosurg Psychiatry. 2008;79(6):664-71.

167. Fokke C, et al. Diagnosis of Guillain-Barre syndrome and validation of Brighton criteria. Brain. 2014;137. (Pt 1:33-43.

168. Wijdicks EF, Klein CJ. Guillain-Barre syndrome. Mayo Clin Proc. 2017;92(3):467-79.

169. Burns TM, et al. Adynamic ileus in severe GuillainBarre syndrome. Muscle Nerve. 2001;24(7):963-5.

170. Emmons PR, Blume WT, DuShane JW. Cardiac monitoring and demand pacemaker in GuillainBarre syndrome. Arch Neurol. 1975;32(1):59-61.

171. Fugate JE, et al. One thing leads to another: GBS complicated by PRES and Takotsubo cardiomyopathy. Neurocrit Care. 2009;11(3):395-7.

172. Lichtenfeld P. Autonomic dysfunction in the GuillainBarre syndrome. Am J Med. 1971;50(6):772-80.
173. Funakoshi K, et al. Clinical predictors of mechanical ventilation in fisher/Guillain-Barre overlap syndrome. J Neurol Neurosurg Psychiatry. 2009; 80(1):60-4.

174. Baraba R, et al. Electrophysiological findings in early Guillain-Barre syndrome. Acta Clin Croat. 2011;50(2):201-7.

175. Vucic S, et al. Neurophysiologic findings in early acute inflammatory demyelinating polyradiculoneuropathy. Clin Neurophysiol. 2004;115(10):2329-35.

176. Kokubun N, et al. Conduction block in acute motor axonal neuropathy. Brain. 2010;133(10):2897-908.

177. Kokubun N, et al. Reversible conduction failure is distinct from neurophysiological patterns of recovery in mild demyelinating Guillain-Barre syndrome. J Neurol Sci. 2013;326(1-2):111-4.

178. Albers JW, Donofrio PD, McGonagle TK. Sequential electrodiagnostic abnormalities in acute inflammatory demyelinating polyradiculoneuropathy. Muscle Nerve. 1985;8(6):528-39.

179. Hughes RA, Swan AV, van Doorn PA. Intravenous immunoglobulin for Guillain-Barre syndrome. Cochrane Database Syst Rev. 2014;9:CD002063.

180. Raphael JC, et al. Plasma exchange for GuillainBarre syndrome. Cochrane Database Syst Rev. 2012;7:CD001798.

181. Efficiency of plasma exchange in Guillain-Barre syndrome: role of replacement fluids. French cooperative group on plasma exchange in Guillain-Barre syndrome. Ann Neurol. 1987;22(6):753-61.

182. Plasmapheresis and acute Guillain-Barre syndrome. The Guillain-Barre syndrome study group. Neurology. 1985;35(8):1096-104.

183. van der Meche FG, Schmitz PI. A randomized trial comparing intravenous immune globulin and plasma exchange in Guillain-Barre syndrome. Dutch Guillain-Barre study group. N Engl J Med. 1992;326(17):1123-9.

184. Plasma Exchange/Sandoglobulin Guillain-Barre Syndrome Trial Group. Randomised trial of plasma exchange, intravenous immunoglobulin, and combined treatments in Guillain-Barre syndrome. Lancet. 1997;349(9047):225-30.

185. Bucelli R, Harms MB. Neuromuscular Emergencies. Semin Neurol. 2015;35(6):683-9.

186. van Koningsveld R, et al. Effect of methylprednisolone when added to standard treatment with intravenous immunoglobulin for Guillain-Barre syndrome: randomised trial. Lancet. 2004;363(9404): 192-6.

187. Rajabally YA, Uncini A. Outcome and its predictors in Guillain-Barre syndrome. J Neurol Neurosurg Psychiatry. 2012;83(7):711-8.

188. Walgaard C, et al. Early recognition of poor prognosis in Guillain-Barre syndrome. Neurology. 2011;76(11):968-75.

189. van Koningsveld R, et al. A clinical prognostic scoring system for Guillain-Barre syndrome. Lancet Neurol. 2007;6(7):589-94. 
190. Drenthen J, et al. Residual fatigue in Guillain-Barre syndrome is related to axonal loss. Neurology. 2013;81(21):1827-31.

191. Merkies IS, Faber CG. Fatigue in immune-mediated neuropathies. Neuromuscul Disord. 2012;22(Suppl 3):S203-7.

192. van den Berg B, et al. Mortality in Guillain-Barre syndrome. Neurology. 2013;80(18):1650-4.

193. Galban-Horcajo F, et al. The application of glycosphingolipid arrays to autoantibody detection in neuroimmunological disorders. Curr Opin Chem Biol. 2014;18:78-86.

194. Rabinstein AA. Noninvasive ventilation for neuromuscular respiratory failure: when to use and when to avoid. Curr Opin Crit Care. 2016;22(2):94-9.

195. Cabrera Serrano M, Rabinstein AA. Causes and outcomes of acute neuromuscular respiratory failure. Arch Neurol. 2010;67(9):1089-94.

196. Rabinstein AA, Wijdicks EF. Warning signs of imminent respiratory failure in neurological patients. Semin Neurol. 2003;23(1):97-104.

197. Lawn ND, et al. Anticipating mechanical ventilation in Guillain-Barre syndrome. Arch Neurol. 2001;58(6):893-8.

198. Nava S, Hill N. Non-invasive ventilation in acute respiratory failure. Lancet. 2009;374(9685):250-9.

199. Rabinstein A, Wijdicks EF. BiPAP in acute respiratory failure due to myasthenic crisis may prevent intubation. Neurology. 2002;59(10):1647-9.

200. Seneviratne J, et al. Noninvasive ventilation in myasthenic crisis. Arch Neurol. 2008;65(1):54-8.

201. Bach JR, et al. Extubation of patients with neuromuscular weakness: a new management paradigm. Chest. 2010;137(5):1033-9.

202. Wijdicks EF, Roy TKl. BiPAP in early guillainBarre syndrome may fail. Can J Neurol Sci. 2006;33(1):105-6.

203. Radunovic A, et al. Mechanical ventilation for amyotrophic lateral sclerosis/motor neuron disease. Cochrane Database Syst Rev. 2013;3:CD004427.

204. Villanova M, Brancalion B, Mehta AD. Duchenne muscular dystrophy: life prolongation by noninvasive ventilatory support. Am J Phys Med Rehabil. 2014;93(7):595-9.

205. Ferguson IT, Murphy RP, Lascelles RG. Ventilatory failure in myasthenia gravis. J Neurol Neurosurg Psychiatry. 1982;45(3):217-22.

206. Klingler W, Lehmann-Horn F, Jurkat-Rott $\mathrm{K}$. Complications of anaesthesia in neuromuscular disorders. Neuromuscul Disord. 2005;15(3): 195-206.

207. Vincken W, Elleker MG, Cosio MG. Determinants of respiratory muscle weakness in stable chronic neuromuscular disorders. Am J Med. 1987; 82(1):53-8.

208. Gozal D. Pulmonary manifestations of neuromuscular disease with special reference to Duchenne muscular dystrophy and spinal muscular atrophy. Pediatr Pulmonol. 2000;29(2):141-50.
209. Racca F, et al. Respiratory management of acute respiratory failure in neuromuscular diseases. Minerva Anestesiol. 2010;76(1):51-62.

210. Blichfeldt-Lauridsen L, Hansen BD. Anesthesia and myasthenia gravis. Acta Anaesthesiol Scand. 2012;56(1):17-22.

211. Wang $\mathrm{CH}$, et al. Consensus statement on standard of care for congenital muscular dystrophies. J Child Neurol. 2010;25(12):1559-81.

212. Rubino FA. Perioperative management of patients with neurologic disease. Neurol Clin. 2004;22(2):261-76.

213. Birnkrant DJ, et al. American College of Chest Physicians consensus statement on the respiratory and related management of patients with Duchenne muscular dystrophy undergoing anesthesia or sedation. Chest. 2007;132(6):1977-86.

214. Birnkrant DJ. The American College of Chest Physicians consensus statement on the respiratory and related management of patients with Duchenne muscular dystrophy undergoing anesthesia or sedation. Pediatrics. 2009;123(Suppl 4): S242-4.

215. Graham RJ, et al. Anesthesia and perioperative medical management of children with spinal muscular atrophy. Paediatr Anaesth. 2009;19(11):1054-63.

216. Muenster T, et al. Anaesthetic management in patients with Duchenne muscular dystrophy undergoing orthopaedic surgery: a review of 232 cases. Eur J Anaesthesiol. 2012;29(10):489-94.

217. Schmitt HJ, Muenster T. Anesthesia in patients with neuromuscular disorders. Minerva Anestesiol. 2009;75(11):632-7.

218. Bushby K, et al. Diagnosis and management of Duchenne muscular dystrophy, part 1: diagnosis, and pharmacological and psychosocial management. Lancet Neurol. 2010;9(1):77-93.

219. Bushby K, et al. Diagnosis and management of Duchenne muscular dystrophy, part 2: implementation of multidisciplinary care. Lancet Neurol. 2010;9(2):177-89.

220. Perloff JK, de Leon AC Jr, O'Doherty D. The cardiomyopathy of progressive muscular dystrophy. Circulation. 1966;33(4):625-48.

221. Sofocleous CT, et al. Sonographically guided placement of peripherally inserted central venous catheters: review of 355 procedures. AJR Am J Roentgenol. 1998;170(6):1613-6.

222. Richa FC. Anaesthetic management of a patient with limb-girdle muscular dystrophy for laparoscopic cholecystectomy. Eur J Anaesthesiol. 2011;28(1):72-3.

223. Lee D, et al. Total intravenous anesthesia without muscle relaxant in a patient with amyotrophic lateral sclerosis. J Anesth. 2008;22(4):443-5.

224. Bach JR, Saporito LR. Criteria for extubation and tracheostomy tube removal for patients with ventilatory failure. A different approach to weaning. Chest. 1996;110(6):1566-71. 
225. de Boer HD, et al. Reversal of rocuronium-induced (1.2 $\mathrm{mg} / \mathrm{kg}$ ) profound neuromuscular block by sugammadex: a multicenter, dose-finding and safety study. Anesthesiology. 2007;107(2):239-44.

226. Unterbuchner C, Fink H, Blobner M. The use of sugammadex in a patient with myasthenia gravis. Anaesthesia. 2010;65(3):302-5.

227. Walker KJ, et al. Ultrasound guidance for peripheral nerve blockade. Cochrane Database Syst Rev. 2009;4:Cd006459.

228. Wang $\mathrm{CH}$, et al. Consensus statement for standard of care in spinal muscular atrophy. J Child Neurol. 2007;22(8):1027-49.
229. Kelly DJ, Ahmad M, Brull SJ. Preemptive analgesia I: physiological pathways and pharmacological modalities. Can J Anaesth. 2001;48(10):1000-10.

230. Hidalgo MP, et al. The clinical effect of small oral clonidine doses on perioperative outcomes in patients undergoing abdominal hysterectomy. Anesth Analg. 2005;100(3):795-802.

231. Niranjan V, Bach JR. Noninvasive management of pediatric neuromuscular ventilatory failure. Crit Care Med. 1998;26(12):2061-5. 